\title{
Critical Set of the Master Function and Characteristic Variety of the Associated Gauss-Manin Differential Equations
}

\section{A. Varchenko ${ }^{1}$}

\begin{abstract}
We consider a weighted family of $n$ parallelly transported hyperplanes in a $k$-dimensional affine space and describe the characteristic variety of the Gauss-Manin differential equations for associated hypergeometric integrals. The characteristic variety is given as the zero set of Laurent polynomials, whose coefficients are determined by weights and the associated point in the Grassmannian $\operatorname{Gr}(k, n)$. The Laurent polynomials are in involution. These statements generalize (Varchenko, Mathematics 2:218-231,2014), where such a description was obtained for a weighted generic family of parallelly transported hyperplanes. An intermediate object between the differential equations and the characteristic variety is the algebra of functions on the critical set of the associated master function. We construct a linear isomorphism between the vector space of the Gauss-Manin differential equations and the algebra of functions. The isomorphism allows us to describe the characteristic variety. It also allowed us to define an integral structure on the vector space of the algebra and the associated (combinatorial) connection on the family of such algebras.
\end{abstract}

Keywords Master functions - Gauss-Manin differential equations · characteristic variety $\cdot$ Hessian and Jacobian $\cdot$ Grothendieck residue

\section{Contents}

1 Introduction . . . . . . . . . . . . . . . . . . . . . . . . . 254

2 Arrangements . . . . . . . . . . . . . . . . . . . . . . . 257

A. Varchenko supported in part by NSF Grant DMS-1362924.

A. Varchenko

anv@email.unc.edu

1 Department of Mathematics, University of North Carolina at Chapel Hill,

Chapel Hill, NC 27599-3250, USA 
2.1 Affine Arrangement . . . . . . . . . . . . . . . . . . . . . . . . . . . . . . . . . . . . . . . . . . . 257

2.2 Orlik-Solomon Algebra . . . . . . . . . . . . . . . . . . . . . . . 257

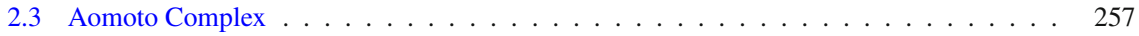

2.4 Flag Complex . . . . . . . . . . . . . . . . . . . . . . . . . . . . . . . . . . . . . . . . . . . . . . . . . . . . . . . . .

2.5 Euler Characteristic of $U(\mathcal{C}) \ldots \ldots \ldots \ldots \ldots \ldots \ldots \ldots$

2.6 Duality. . . . . . . . . . . . . . . . . . . . . . . . 258

2.7 Contravariant Map and Form . . . . . . . . . . . . . . . . . . . . . . . . . . . . 259

2.8 Generic Weights . . . . . . . . . . . . . . . . . . . . . . . . . 259

2.9 Differential Forms . . . . . . . . . . . . . . . . . . . . . . . . . . . . . . 259

2.10 Master Function . . . . . . . . . . . . . . . . . . . . . . . . . . . . . . . . . . . . . . . . . . . . . . . . . . . .

2.11 Isolated Critical Points . . . . . . . . . . . . . . . . . . . . . . . . . . . . 260

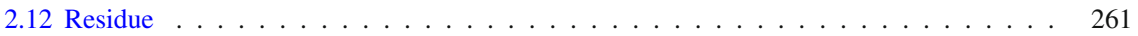

2.13 Canonical Element . . . . . . . . . . . . . . . . . . . . . . . . . 261

2.14 Canonical Isomorphism . . . . . . . . . . . . . . . . . . . . . . . . . . . 262

2.15 Orthogonal Projection . . . . . . . . . . . . . . . . . . . . . 263

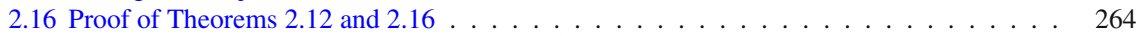

2.17 Integral Structure on $\mathcal{O}\left(C_{\mathcal{C}, a}\right)$ and $\operatorname{Sing}_{a} \mathcal{F}^{k}(\mathcal{C}) \ldots \ldots \ldots \ldots \ldots$

2.18 Skew-Commutative Versus Commutative . . . . . . . . . . . . . . . . . . . 266

2.19 Combinatorial Connection . . . . . . . . . . . . . . . . . . . 266

2.20 Arrangement with Normal Crossings . . . . . . . . . . . . . . . . . . . . . . . 266

3 Family of Parallelly Transported Hyperplanes . . . . . . . . . . . . . . . . . . . . . . . . . 267

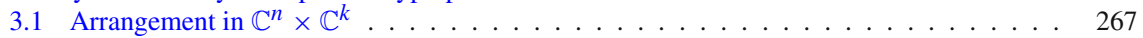

3.2 Discriminant . . . . . . . . . . . . . . . . . . . . . . . 268

3.3 Combinatorial Connection . . . . . . . . . . . . . . . . . . . 268

3.4 Operators $K_{j} \in \mathcal{O}\left(\mathbb{C}^{n}-\Delta\right) \otimes($ End $V), j \in J \ldots \ldots \ldots \ldots$

3.5 Corollary of Theorem $3.3 \ldots \ldots \ldots \ldots$. . . . . . . . . . . . . . 270

3.6 Gauss-Manin Connection on $\left(\mathbb{C}^{n}-\Delta\right) \times\left(\operatorname{Sing}_{a} V\right) \rightarrow \mathbb{C}^{n}-\Delta \ldots \ldots 270$

3.7 Critical Set . . . . . . . . . . . . . . . . . . . . . . 271

3.8 Formulas for Multiplication . . . . . . . . . . . . . . . . . . . . 273

3.9 Corollary of Theorem $3.7 \ldots \ldots \ldots \ldots \ldots \ldots$. . . . . . . . . . . . . . . . . . . . . . . . . . . . . . . . . . 273

4 Langrangian Variety and Critical Set . . . . . . . . . . . . . . . . . . . . . . . . . . . . 274

4.1 Lagrangian Variety . . . . . . . . . . . . . . . . . . . . . . . . . . . . . . . . . . . . . . . . . . . . . . . . . . . . .

4.2 Fibers of $\pi_{L_{Y, a}} \ldots \ldots \ldots \ldots \ldots \ldots \ldots \ldots \ldots \ldots$

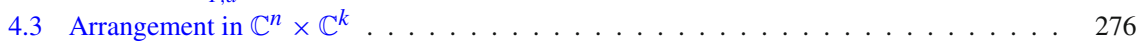

4.4 Hessian and Jacobian . . . . . . . . . . . . . . . . . . . . . . . . . . . . . 278

4.5 Corollaries of Theorem $4.5 \ldots \ldots \ldots \ldots \ldots \ldots \ldots \ldots$

4.6 Real Solutions . . . . . . . . . . . . . . . . . . . . . . . . . . 280

5 Characteristic Variety of the Gauss-Manin Differential Equations . . . . . . . . . . . . . . . 280

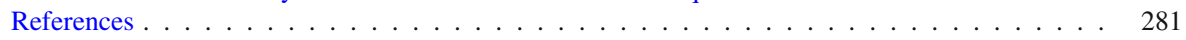

\section{Introduction}

There are three places, where a flat connection depending on a parameter appears:

- KZ equations, $\kappa \frac{\partial I}{\partial z_{i}}=K_{i} I, i=1, \ldots, n$. Here $\kappa$ is a parameter, $I\left(z_{1}, \ldots, z_{n}\right)$ a $V$ valued function, where $V$ is a vector space from representation theory, $K_{i}: V \rightarrow V$ are linear operators, depending on $z$. The connection is flat for all $\kappa$, see, for example, Etingof et al. (1998), Varchenko (1995).

- Quantum differential equations, $\kappa \frac{\partial I}{\partial z_{i}}=p_{i} *_{z} I, i=1, \ldots, n$. Here $p_{1}, \ldots, p_{n}$ are generators of some commutative algebra $H$ with quantum multiplication $*_{z}$ depending on $z$. The connection is flat for all $\kappa$. These equations are a part of the Frobenius structure on the quantum cohomology of a variety, see Dubrovin (1996), Manin (1999). 
- Differential equations for hypergeometric integrals associated with a family of weighted arrangements with parallelly transported hyperplanes, $\kappa \frac{\partial I}{\partial z_{i}}=K_{i} I, i=$ $1, \ldots, n$. The connection is flat for all $\kappa$, see, for example, Varchenko (1989), Orlik and Terao (2001).

If $\kappa \frac{\partial I}{\partial z_{i}}=K_{i} I, i=1, \ldots, n$, is a system of $V$-valued differential equations of one of these types, then its characteristic variety is

$$
\text { Spec }=\left\{(q, p) \in T^{*} \mathbb{C}^{n} \mid \exists v \in V-\{0\} \text { with } K_{j}(q) v=p_{j} v, j=1, \ldots, n\right\} .
$$

It is known that the characteristic varieties of the first two types of differential equation are interesting objects. For example, the characteristic variety of the quantum differential equation of the flag variety is the zero set of the Hamiltonians of the classical Toda lattice, see Givental (1997), Givental and Kim (1995), and the characteristic variety of the $\mathfrak{g l}_{N} \mathrm{KZ}$ equations with values in the tensor power of the vector representation is the zero set of the Hamiltonians of the classical Calogero-Moser system, see Mukhin et al. (2012).

In this paper we describe the characteristic variety of the Gauss-Manin differential equations for hypergeometric integrals associated with an arbitrary weighted family of $n$ parallelly transported hyperplanes in $\mathbb{C}^{k}$. This description generalizes (Varchenko 2014, Corollary 4.2) where such a description was obtained for a weighted generic family of parallelly transported hyperplanes.

The characteristic variety is given as the zero set of Laurent polynomials, whose coefficients are determined by weights and the associated point in the Grassmannian $\operatorname{Gr}(k, n)$. The Laurent polynomials are in involution, see Sect. 4.1.

It is known that the KZ differential equations, as well as some quantum differential equations, can be identified with certain symmetric parts of the Gauss-Manin differential equations of weighted families of parallelly transported hyperplanes, see Schechtman and Varchenko (1991), Tarasov and Varchenko (2014). Therefore, the results of this paper on the characteristic variety is a step to studying characteristic varieties of more general $\mathrm{KZ}$ and quantum differential equations, which admit integral hypergeometric representations.

Our description of the characteristic variety is based on the fact (Varchenko 2006), that the characteristic variety of the Gauss-Manin differential equations is generated by the master function of the corresponding hypergeometric integrals, that is, the characteristic variety coincides with the Lagrangian variety of the master function. That fact was developed later in Mukhin et al. (2011, Theorem 5.5), it was proved there with the help of the Bethe ansatz, that the local algebra of a critical point of the master function associated with a $\mathfrak{g l}_{N} \mathrm{KZ}$ equation can be identified with a suitable local Bethe algebra of the corresponding $\mathfrak{g l}_{N}$ module.

In Sect. 2, we consider a weighted arrangement $(\mathcal{C}, a)$ of $n$ affine hyperplanes in $\mathbb{C}^{k}$. Here $a$ is a point of $\left(\mathbb{C}^{\times}\right)^{n}$ called the weight. We introduce the Aomoto complex $\left(\mathcal{A}(\mathcal{C}), d^{(a)}\right)$, the flag complex $(\mathcal{F}(\mathcal{C}), d)$, the critical set $C_{\mathcal{C}, a}$ of the master function on the complement to the arrangement. We remind the isomorphism of vector spaces $\mathcal{E}: \mathcal{O}\left(C_{\mathcal{C}, a}\right) \rightarrow H^{k}(\mathcal{F}(\mathcal{C}), d)$, constructed in Varchenko (2011) and given by a variant of the Grothendieck residue. The algebra $\mathcal{O}\left(C_{\mathcal{C}, a}\right)$ is a nonlinear object, defined by the critical point equations; the space $H^{k}(\mathcal{F}(\mathcal{C}), d)$ is a combinatorial object defined 
by the matroid of the arrangement $\mathcal{C}$; the isomorphism $\mathcal{E}$ is given by a $k$-dimensional integral. Our first main result, Theorem 2.16, gives an elementary isomorphism $\left[\mathcal{S}^{(a)}\right]$ : $H^{k}(\mathcal{F}(\mathcal{C}), d) \rightarrow \mathcal{O}\left(C_{\mathcal{C}, a}\right)$ such that $\left[\mathcal{S}^{(a)}\right] \circ \mathcal{E}=(-1)^{k}$. Theorem 2.16 allows us to bring to $\mathcal{O}\left(C_{\mathcal{C}, a}\right)$ the combinatorial structures on $H^{k}(\mathcal{F}(\mathcal{C}), d)$. In particular, we construct a set $\left\{w_{\alpha_{0}, \ldots, \alpha_{k}}\right\}$ of marked elements of $\mathcal{O}\left(C_{\mathcal{C}, a}\right)$, labeled by flags of edges of $\mathcal{C}$, which spans the vector space $\mathcal{O}\left(C_{\mathcal{C}, a}\right)$. We give the linear relations between the marked elements; the relations are with integer coefficients and depend only on the matroid of $\mathcal{C}$, see Corollary 2.18.

In Sect. 3, we consider a family of weighted arrangements $(\mathcal{C}(x), a)$ of $n$ affine hyperplanes in $\mathbb{C}^{k}$, parameterized by $x \in \mathbb{C}^{n}$. The hyperplanes of $(\mathcal{C}(x), a)$ are transported parallelly as $x$ changes. Each of the arrangements has the algebra $\mathcal{O}\left(C_{\mathcal{C}(x), a}\right)$ of functions on the critical set $C_{\mathcal{C}(x), a}$ of the associated master function. We define the discriminant $\Delta \subset \mathbb{C}^{n}$ so that the combinatorics of $\mathcal{C}(x)$ does not change when $x$ runs through $\mathbb{C}^{n}-\Delta$. The constructions of Sect. 2 provide us with the vector bundle of algebras $\sqcup_{x \in \mathbb{C}^{n}-\Delta} \mathcal{O}\left(C_{\mathcal{C}(x), a}\right) \rightarrow \mathbb{C}^{n}-\Delta$, whose fibers are canonically identified with the help of the marked elements. The multiplication in $\mathcal{O}\left(C_{\mathcal{C}}(x), a\right)$ depends on $x$. The isomorphism $H^{k}(\mathcal{F}(\mathcal{C}(x)), d) \rightarrow \mathcal{O}\left(C_{\mathcal{C}(x), a}\right)$ of Sect. 2 allows us to describe the multiplication in $\mathcal{O}\left(C_{\mathcal{C}(x), a}\right)$ combinatorially, see Corollary 3.8. We describe the Gauss-Manin differential equations associated with the weighted family of arrangements as a system of differential equations on the bundle of algebras.

In Sect. 4.1, for given $k$-dimensional vector subspace $Y \subset \mathbb{C}^{n}$ and weight $a \in\left(\mathbb{C}^{\times}\right)^{n}$, we define a Lagrangian variety $L_{Y, a} \subset \mathbb{C}^{n} \times\left(\mathbb{C}^{n}\right)^{*}$. We consider the projection $\pi_{L_{Y, a}}: L_{Y, a} \rightarrow \mathbb{C}^{n}$ and the algebras of functions $\mathcal{O}\left(L_{Y, a}(x)\right)$ on fibers of the projection. We describe $L_{Y, a}$ as the zero set of Laurent polynomials in involution.

In Sect. 4.3, for the family of arrangements $\mathcal{C}(x)$ considered in Sect. 3, we define a $k$-dimensional subspace $Y \subset \mathbb{C}^{n}$ and construct an isomorphism $\Psi_{\mathcal{C}(x), a}^{*}$ : $\mathcal{O}\left(L_{Y, a}(x)\right) \rightarrow \mathcal{O}\left(C_{\mathcal{C}(x), a}\right)$ of algebras for any $x \in \mathbb{C}^{n}$. Theorem 4.5 , on this isomorphism, is our second main result. We discuss corollaries of Theorem 4.5 in Sects. 4.4, 4.5 and 4.6. In particular, in Corollary 4.10 we describe the ratio of the Hessian element in $\mathcal{O}\left(C_{\mathcal{C}(x), a}\right)$ and the Jacobian element in $\mathcal{O}\left(L_{Y, a}(x)\right)$ and in Corollary 4.11 we identify the standard residue form on $\mathcal{O}\left(C_{\mathcal{C}(x), a}\right)$, defined by a $k$-dimensional integral, with a residue form on $\mathcal{O}\left(L_{Y, a}(x)\right)$, defined by an $n$-dimensional integral. In Sect. 4.5, we consider the vector bundle of algebras $\sqcup_{x \in \mathbb{C}^{n}-\Delta} \mathcal{O}\left(L_{Y, a}(x)\right) \rightarrow \mathbb{C}^{n}-\Delta$. The isomorphism $\Psi_{\mathcal{C}(x), a}^{*}$ allows us to identify the fibers of the bundle and describe the Gauss-Manin differential equations with values in that bundle. They have the form $\kappa \frac{\partial I}{\partial q_{j}}(x)=\left[p_{j}\right] *_{x} I, j=1, \ldots, n$, where $q_{1}, \ldots, q_{n}$ are coordinates on $\mathbb{C}^{n}$, $p_{1}, \ldots, p_{n}$ are the dual coordinates on $\left(\mathbb{C}^{n}\right)^{*},\left[p_{j}\right] *_{x}$ is the multiplication by $p_{j}$ in $\mathcal{O}\left(L_{Y, a}(x)\right)$.

In Sect. 4.6, we observe a rather unexpected 'reality' property of the Lagrangian variety $L_{Y, a}$, which is similar to the reality property of Schubert calculus, see Mukhin et al. (2009a, b), Sottile (2010).

In Theorem 5.1, we identify the characteristic variety of the Gauss-Manin differential equations associated with the family of arrangements considered in Sect. 3 and the Lagrangian variety $L_{Y, a}$ defined in Sect. 4.3. 


\section{Arrangements}

\subsection{Affine Arrangement}

Let $k, n$ be positive integers, $k<n$. Denote $J=\{1, \ldots, n\}$.

Consider the complex affine space $\mathbb{C}^{k}$ with coordinates $t_{1}, \ldots, t_{k}$. Let $\mathcal{C}=\left(H_{j}\right)_{j \in J}$, be an arrangement of $n$ affine hyperplanes in $\mathbb{C}^{k}$. Denote $U(\mathcal{C})=\mathbb{C}^{k}-\cup_{j \in J} H_{j}$, the complement. An edge $X_{\alpha} \subset \mathbb{C}^{k}$ of $\mathcal{C}$ is a nonempty intersection of some hyperplanes of $\mathcal{C}$. Denote by $J_{\alpha} \subset J$ the subset of indices of all hyperplanes containing $X_{\alpha}$. Denote $l_{\alpha}=\operatorname{codim}_{\mathbb{C}^{k}} X_{\alpha}$.

We assume that $\mathcal{C}$ is essential, that is, $\mathcal{C}$ has a vertex, an edge which is a point.

An edge is called dense if the subarrangement of all hyperplanes containing it is irreducible: the hyperplanes cannot be partitioned into nonempty sets so that, after a change of coordinates, hyperplanes in different sets are in different coordinates. In particular, each hyperplane of $\mathcal{C}$ is a dense edge.

\subsection{Orlik-Solomon Algebra}

Define complex vector spaces $\mathcal{A}^{p}(\mathcal{C}), p=0, \ldots, k$. For $p=0$, we set $\mathcal{A}^{p}(\mathcal{C})=\mathbb{C}$. For $p \geq 1, \mathcal{A}^{p}(\mathcal{C})$ is generated by symbols $\left(H_{j_{1}}, \ldots, H_{j_{p}}\right)$ with $j_{i} \in J$, such that

(i) $\left(H_{j_{1}}, \ldots, H_{j_{p}}\right)=0$ if $H_{j_{1}}, \ldots, H_{j_{p}}$ are not in general position, that is, if the intersection $H_{j_{1}} \cap \cdots \cap H_{j_{p}}$ is empty or has codimension less than $p$;

(ii) $\left(H_{j_{\sigma(1)}}, \ldots, H_{j_{\sigma(p)}}\right)=(-1)^{|\sigma|}\left(H_{j_{1}}, \ldots, H_{j_{p}}\right)$ for any element $\sigma$ of the symmetric group $\Sigma_{p}$

(iii) $\sum_{i=1}^{p+1}(-1)^{i}\left(H_{j_{1}}, \ldots, \widehat{H}_{j_{i}}, \ldots, H_{j_{p+1}}\right)=0$ for any $(p+1)$-tuple $H_{j_{1}}, \ldots, H_{j_{p+1}}$ of hyperplanes in $\mathcal{C}$ which are not in general position and such that $H_{j_{1}} \cap \cdots \cap$ $H_{j_{p+1}} \neq \emptyset$.

The direct sum $\mathcal{A}(\mathcal{C})=\oplus_{p=1}^{N} \mathcal{A}^{p}(\mathcal{C})$ is the Orlik-Solomon algebra with respect to multiplication $\left(H_{j_{1}}, \ldots, H_{j_{p}}\right) \cdot\left(H_{j_{p+1}}, \ldots, H_{j_{p+q}}\right)=\left(H_{j_{1}}, \ldots, H_{j_{p}}, H_{j_{p+1}}, \ldots, H_{j_{p+q}}\right)$.

\subsection{Aomoto Complex}

Fix a point $a=\left(a_{1}, \ldots, a_{n}\right) \in\left(\mathbb{C}^{\times}\right)^{n}$ called the weight. Then the arrangement $\mathcal{C}$ is weighted: for $j \in J$, we assign weight $a_{j}$ to hyperplane $H_{j}$. For an edge $X_{\alpha}$, define its weight $a_{\alpha}=\sum_{j \in J_{\alpha}} a_{j}$. We define $\omega^{(a)}=\sum_{j \in J} a_{j} \cdot\left(H_{j}\right) \in \mathcal{A}^{1}(\mathcal{C})$. Multiplication by $\omega^{(a)}$ defines the differential $d^{(a)}: \mathcal{A}^{p}(\mathcal{C}) \rightarrow \mathcal{A}^{p+1}(\mathcal{C}), x \mapsto \omega^{(a)} \cdot x$, on $\mathcal{A}(\mathcal{C})$, $\left(d^{(a)}\right)^{2}=0$. The complex $\left(\mathcal{A}(\mathcal{C}), d^{(a)}\right)$ is called the Aomoto complex.

\subsection{Flag Complex}

For an edge $X_{\alpha}, l_{\alpha}=p$, a flag starting at $X_{\alpha}$ is a sequence $X_{\alpha_{0}} \supset X_{\alpha_{1}} \supset \ldots \supset$ $X_{\alpha_{p}}=X_{\alpha}$ of edges such that $l_{\alpha_{j}}=j$ for $j=0, \ldots, p$. For an edge $X_{\alpha}$, we define $\left(\overline{\mathcal{F}}_{\alpha}\right)_{\mathbb{Z}}$ as the free $\mathbb{Z}$-module generated by the elements $\bar{F}_{\alpha_{0}, \ldots, \alpha_{p}=\alpha}$ labeled by the 
elements of the set of all flags starting at $X_{\alpha}$. We define $\left(\mathcal{F}_{\alpha}\right)_{\mathbb{Z}}$ as the quotient of $\left(\overline{\mathcal{F}}_{\alpha}\right)_{\mathbb{Z}}$ by the submodule generated by all the elements of the form

$$
\sum_{X_{\beta}, X_{\alpha_{j-1}} \supset X_{\beta} \supset X_{\alpha_{j+1}}} \bar{F}_{\alpha_{0}, \ldots, \alpha_{j-1}, \beta, \alpha_{j+1}, \ldots, \alpha_{p}=\alpha}
$$

Such an element is determined by $j \in\{1, \ldots, p-1\}$ and an incomplete flag $X_{\alpha_{0}} \supset$ $\cdots \supset X_{\alpha_{j-1}} \supset X_{\alpha_{j+1}} \supset \cdots \supset X_{\alpha_{p}}=X_{\alpha}$ with $l_{\alpha_{i}}=i$.

We denote by $F_{\alpha_{0}, \ldots, \alpha_{p}}$ the image in $\left(\mathcal{F}_{\alpha}\right)_{\mathbb{Z}}$ of the element $\bar{F}_{\alpha_{0}, \ldots, \alpha_{p}}$. For $p=$ $0, \ldots, k$, we set $\left(\mathcal{F}^{p}(\mathcal{C})\right)_{\mathbb{Z}}=\oplus_{X_{\alpha}, l_{\alpha}=p}\left(\mathcal{F}_{\alpha}\right)_{\mathbb{Z}}, \mathcal{F}^{p}(\mathcal{C})=\left(\mathcal{F}^{p}(\mathcal{C})\right)_{\mathbb{Z}} \otimes \mathbb{C}, \mathcal{F}(\mathcal{C})=$ $\oplus_{p=1}^{N} \mathcal{F}^{p}(\mathcal{C})$. We define the differential $d_{\mathbb{Z}}:\left(\mathcal{F}^{p}(\mathcal{C})\right)_{\mathbb{Z}} \rightarrow\left(\mathcal{F}^{p+1}(\mathcal{C})\right)_{\mathbb{Z}}$ by

$$
d_{\mathbb{Z}}: F_{\alpha_{0}, \ldots, \alpha_{p}} \mapsto \sum_{X_{\beta}, X_{\alpha_{p}} \supset X_{\beta}} F_{\alpha_{0}, \ldots, \alpha_{p}, \beta},
$$

$d_{\mathbb{Z}}^{2}=0$. Tensoring $d_{\mathbb{Z}}$ with $\mathbb{C}$, we obtain the differential $d: \mathcal{F}^{p}(\mathcal{C}) \rightarrow \mathcal{F}^{p+1}(\mathcal{C})$. In particular, we have (see Schechtman and Varchenko 1991)

$$
H^{p}(\mathcal{F}(\mathcal{C}), d)=H^{p}\left((\mathcal{F}(\mathcal{C}))_{\mathbb{Z}}, d_{\mathbb{Z}}\right) \otimes \mathbb{C}
$$

Theorem 2.1 (Schechtman and Varchenko 1991, Corollary 2.8) We have $H^{p}(\mathcal{F}(\mathcal{C}), d)=0$ for $p \neq k$ and $\operatorname{dim} H^{k}(\mathcal{F}(\mathcal{C}), d)=|\chi(U(\mathcal{C}))|$, where $\chi(U(\mathcal{C}))$ is the Euler characteristic of the complement $U(\mathcal{C})$.

\subsection{Euler Characteristic of $U(\mathcal{C})$}

A formula for the Euler characteristic $\chi(U(\mathcal{C}))$ in terms of the matroid associated with $\mathcal{C}$ is given in Schechtman et al. (1995, Proposition 2.3). The condition $\chi(U(\mathcal{C})) \neq 0$ is discussed in Crapo (1967, Theorem 2) cited as Theorem 2.4 in Schechtman et al. (1995). On the equality of the absolute value $|\chi(U(\mathcal{C}))|$ and the number of bounded components of the real part of $U(\mathcal{C})$ see, for example, Varchenko (1995).

\subsection{Duality}

The vector spaces $\mathcal{A}^{p}(\mathcal{C})$ and $\mathcal{F}^{p}(\mathcal{C})$ are dual, see Schechtman and Varchenko (1991). The pairing $\mathcal{A}^{p}(\mathcal{C}) \otimes \mathcal{F}^{p}(\mathcal{C}) \rightarrow \mathbb{C}$ is defined as follows. For $H_{j_{1}}, \ldots, H_{j_{p}}$ in general position, set $F\left(H_{j_{1}}, \ldots, H_{j_{p}}\right)=F_{\alpha_{0}, \ldots, \alpha_{p}}$, where $X_{\alpha_{0}}=\mathbb{C}^{k}, X_{\alpha_{1}}=H_{j_{1}}, \ldots, X_{\alpha_{p}}=$ $H_{j_{1}} \cap \cdots \cap H_{j_{p}}$. Then we define $\left\langle\left(H_{j_{1}}, \ldots, H_{j_{p}}\right), F_{\alpha_{0}, \ldots, \alpha_{p}}\right\rangle=(-1)^{|\sigma|}$, if $F_{\alpha_{0}, \ldots, \alpha_{p}}=$ $F\left(H_{j_{\sigma(1)}}, \ldots, H_{j_{\sigma(p)}}\right)$ for some $\sigma \in S_{p}$, and $\left\langle\left(H_{j_{1}}, \ldots, H_{j_{p}}\right), F_{\alpha_{0}, \ldots, \alpha_{p}}\right\rangle=0$ otherwise.

An element $F \in \mathcal{F}^{k}(\mathcal{C})$ is called singular if $F$ annihilates the image of the map $d^{(a)}: \mathcal{A}^{k-1}(\mathcal{C}) \rightarrow \mathcal{A}^{k}(\mathcal{C})$, see Varchenko (2006). Denote by $\operatorname{Sing}_{a} \mathcal{F}^{k}(\mathcal{C}) \subset \mathcal{F}^{k}(\mathcal{C})$ the subspace of all singular vectors. 


\subsection{Contravariant Map and Form}

The weight $a$ determines the contravariant map

$$
\mathcal{S}^{(a)}: \mathcal{F}^{p}(\mathcal{C}) \rightarrow \mathcal{A}^{p}(\mathcal{C}), \quad F_{\alpha_{0}, \ldots, \alpha_{p}} \mapsto \sum a_{j_{1}} \cdots a_{j_{p}}\left(H_{j_{1}}, \ldots, H_{j_{p}}\right)
$$

where the sum is taken over all $p$-tuples $\left(H_{j_{1}}, \ldots, H_{j_{p}}\right)$ such that $H_{j_{1}} \supset X_{\alpha_{1}}, \ldots$, $H_{j_{p}} \supset X_{\alpha_{p}}$. Identifying $\mathcal{A}^{p}(\mathcal{C})$ with $\mathcal{F}^{p}(\mathcal{C})^{*}$, we consider the map as a bilinear form, $S^{(a)}: \mathcal{F}^{p}(\mathcal{C}) \otimes \mathcal{F}^{p}(\mathcal{C}) \rightarrow \mathbb{C}$. The bilinear form is called the contravariant form. The contravariant form is symmetric. For $F_{1}, F_{2} \in \mathcal{F}^{p}(\mathcal{C})$,

$S^{(a)}\left(F_{1}, F_{2}\right)=\sum_{\left\{j_{1}, \ldots, j_{p}\right\} \subset J} a_{j_{1}} \cdots a_{j_{p}}\left\langle\left(H_{j_{1}}, \ldots, H_{j_{p}}\right), F_{1}\right\rangle\left\langle\left(H_{j_{1}}, \ldots, H_{j_{p}}\right), F_{2}\right\rangle$

where the sum is over all unordered $p$-element subsets (see Schechtman and Varchenko 1991).

Lemma 2.2 (Schechtman and Varchenko 1991, Lemma 3.2.5) The contravariant map (2.4) defines a homomorphism of complexes $\mathcal{S}^{(a)}:(\mathcal{F}(\mathcal{C}), d) \rightarrow\left(\mathcal{A}(\mathcal{C}), d^{(a)}\right)$.

\subsection{Generic Weights}

Theorem 2.3 (Schechtman and Varchenko 1991, Theorem 3.7) If the weight a is such that none of the dense edges has weight zero, then the contravariant form is nondegenerate. In particular, we have an isomorphism of complexes $\mathcal{S}:(\mathcal{F}(\mathcal{C}), d) \rightarrow$ $\left(\mathcal{A}(\mathcal{C}), d^{(a)}\right)$.

Theorem 2.4 (Schechtman and Varchenko 1991; Yuzvinsky 1995; Orlik and Terao 2001) If the weight $a$ is such that none of the dense edges has weight zero, then $H^{p}\left(\mathcal{A}^{*}(\mathcal{C}), d^{(a)}\right)=0$ for $p \neq k$ and $\operatorname{dim} H^{k}\left(\mathcal{A}^{*}, d^{(a)}\right)=|\chi(U(\mathcal{C}))|$.

Theorem 2.4 is a corollary of Lemma 2.2 and Theorems 2.1, 2.3.

Corollary 2.5 If the weight $a$ is such that none of the dense edges has weight zero, then the dimension of $\operatorname{Sing}_{a} \mathcal{F}^{k}(\mathcal{C})$ equals $|\chi(U(\mathcal{C}))|$.

Notice that none of the dense edges has weight zero if all weights are positive.

\subsection{Differential Forms}

For $j \in J$, fix defining equations $f_{j}=0$ for the hyperplanes $H_{j}$, where $f_{j}=$ $b_{j}^{1} t_{1}+\cdots+b_{j}^{k} t_{k}+z_{j}$ with $b_{j}^{i}, z_{j} \in \mathbb{C}$. Consider the logarithmic differential 1-form $\omega_{j}=d f_{j} / f_{j}$ on $\mathbb{C}^{k}$. Let $\overline{\mathcal{A}}(\mathcal{C})$ be the exterior $\mathbb{C}$-algebra of differential forms generated by 1 and $\omega_{j}, j \in J$. The map $\mathcal{A}(\mathcal{C}) \rightarrow \overline{\mathcal{A}}(\mathcal{C}),\left(H_{j}\right) \mapsto \omega_{j}$, is an isomorphism. We identify $\mathcal{A}(\mathcal{C})$ and $\overline{\mathcal{A}}(\mathcal{C})$. 
For $I=\left\{i_{1}, \ldots, i_{k}\right\} \subset J$, denote $d_{I}=d_{i_{1}, \ldots, i_{k}}=\operatorname{det}_{i, l=1}^{k}\left(b_{i_{l}}^{i}\right)$. Then

$$
\omega_{i_{1}} \wedge \cdots \wedge \omega_{i_{k}}=\frac{d_{i_{1}, \ldots, i_{k}}}{f_{i_{1}} \ldots f_{i_{k}}} d t_{1} \wedge \cdots \wedge d t_{k}
$$

Lemma 2.6 The functions $\left(1 / f_{j}\right)_{j \in J}$ separate points of $U(\mathcal{C})$.

\subsection{Master Function}

The master function of the weighted arrangement $(\mathcal{C}, a)$ is

$$
\Phi_{\mathcal{C}, a}=\sum_{j \in J} a_{j} \log f_{j}
$$

a multivalued function on $U(\mathcal{C})$. We have $d \Phi_{\mathcal{C}, a}=\sum_{j \in J} a_{j} \omega_{j}=\omega^{(a)} \in \mathcal{A}^{1}(\mathcal{C})$. Let $C_{\mathcal{C}, a}=\left\{u \in U(\mathcal{C}) \mid \frac{\partial \Phi_{\mathcal{C}, a}}{\partial t_{i}}(u)=0\right.$ for $\left.i=1, \ldots, k\right\}$ be the critical set of $\Phi_{\mathcal{C}, a}$. The critical point equations can be reformulated as the equation $\left.\omega^{(a)}\right|_{u}=0$. Notice that

$$
\frac{\partial \Phi_{\mathcal{C}, a}}{\partial t_{i}}=\sum_{j=1}^{n} b_{j}^{i} \frac{a_{j}}{f_{j}} \quad \text { and } \quad \frac{\partial \Phi_{\mathcal{C}, a}}{\partial z_{j}}=\frac{a_{j}}{f_{j}} .
$$

Define the Hessian of the master function, $\operatorname{Hess}_{\mathcal{C}, a}=\operatorname{det}_{i, j=1}^{k}\left(\frac{\partial^{2} \Phi_{\mathcal{C}, a}}{\partial t_{i} \partial t_{j}}\right)$. A critical point $u \in C_{\mathcal{C}, a}$ is nondegenerate if $\operatorname{Hess}_{\mathcal{C}, a}(u) \neq 0$. We have the formula in Varchenko (2006):

$$
\operatorname{Hess}_{\mathcal{C}, a}=(-1)^{k} \sum_{I \subset J,|I|=k} d_{I}^{2} \prod_{i \in I} \frac{a_{i}}{f_{i}^{2}}
$$

\subsection{Isolated Critical Points}

Theorem 2.7 (Varchenko 1995; Orlik and Terao 1995; Silvotti 1996) For generic exponent $a \in\left(\mathbb{C}^{\times}\right)^{n}$, all critical points of $\Phi_{\mathcal{C}, a}$ are nondegenerate and the number of critical points equals $|\chi(U(\mathcal{C}))|$.

Consider the projective space $\mathbb{P}^{k}$ compactifying $\mathbb{C}^{k}$. Assign the weight $a_{\infty}=$ $-\sum_{j \in J} a_{j}$ to the hyperplane $H_{\infty}=\mathbb{P}^{k}-\mathbb{C}^{k}$. Denote by $\mathcal{C}^{\vee}$ the arrangement $\left(H_{j}\right)_{j \in J \cup \infty}$ in $\mathbb{P}^{k}$. The weighted arrangement $(\mathcal{C}, a)$ is called unbalanced if the weight of any dense edge of $\mathcal{C}^{\vee}$ is nonzero, see Varchenko (2011). For example, $(\mathcal{C}, a)$ is unbalanced if all weights $\left(a_{j}\right)_{j \in J}$ are positive. The unbalanced weights form a Zariski open subset in the space of all weights $a \in\left(\mathbb{C}^{\times}\right)^{n}$.

Lemma 2.8 (Varchenko 2011, Section 4) If (C,a) is unbalanced, then all critical points of $\Phi_{\mathcal{C}, a}$ are isolated and the sum of their Milnor numbers equals $|\chi(U(\mathcal{C}))|$. 


\subsection{Residue}

Let $\mathcal{O}(U(\mathcal{C}))$ be the algebra of regular functions on $U(\mathcal{C})$ and $I_{\mathcal{C}, a}=\left\langle\frac{\partial \Phi_{\mathcal{C}, a}}{\partial t_{i}}\right| i=$ $1, \ldots, k\rangle \subset \mathcal{O}(U(\mathcal{C}))$ the ideal generated by the first derivatives of $\Phi_{\mathcal{C}, a}$. Let $\mathcal{O}\left(C_{\mathcal{C}, a}\right)=\mathcal{O}(U(\mathcal{C})) / I_{\mathcal{C}, a}$ be the algebra of functions on the critical set and [] : $\mathcal{O}(U(\mathcal{C})) \rightarrow \mathcal{O}\left(C_{\mathcal{C}, a}\right), f \mapsto[f]$, the projection. The algebra $\mathcal{O}\left(C_{\mathcal{C}, a}\right)$ is finite-dimensional, if all critical points are isolated. In that case, $\mathcal{O}\left(C_{\mathcal{C}, a}\right)=$ $\oplus_{u \in C_{\mathcal{C}, a}} \mathcal{O}\left(C_{\mathcal{C}, a}\right)_{u}$, where $\mathcal{O}\left(C_{\mathcal{C}, a}\right)_{u}$ is the local algebra corresponding to the point $u$.

Lemma 2.9 (Varchenko 2011, Lemma 2.5) If the algebra $\mathcal{O}\left(C_{\mathcal{C}, a}\right)$ is finitedimensional, then the elements $\left[1 / f_{j}\right], j \in J$, generate $\mathcal{O}\left(C_{\mathcal{C}, a}\right)$.

Let $\mathcal{R}_{u}: \mathcal{O}\left(C_{\mathcal{C}, a}\right)_{u} \rightarrow \mathbb{C}$ be the Grothendieck residue,

$$
[f] \mapsto \frac{1}{(2 \pi i)^{k}} \operatorname{Res}_{u} \frac{f}{\prod_{i=1}^{k} \frac{\partial \Phi_{\mathcal{C}, a}}{\partial t_{i}}}=\frac{1}{(2 \pi i)^{k}} \int_{\Gamma_{u}} \frac{f d t_{1} \wedge \cdots \wedge d t_{k}}{\prod_{i=1}^{k} \frac{\partial \Phi_{\mathcal{C}, a}}{\partial t_{i}}}
$$

Here $\Gamma_{u}$ is the real $k$-cycle located in a small neighborhood of $u$ and defined by the equations $\left|\frac{\partial \Phi_{\mathcal{C}, a}}{\partial t_{i}}\right|=\epsilon_{i}, i=1, \ldots, k$, where $\epsilon_{s}$ are sufficiently small positive numbers. The cycle is oriented by the condition $d \arg \frac{\partial \Phi_{\mathcal{C}, a}}{\partial t_{1}} \wedge \cdots \wedge d \arg \frac{\partial \Phi_{\mathcal{C}, a}}{\partial t_{k}}>0$, see Griffiths and Harris (1994).

Denote by $\left[\operatorname{Hess}_{\mathcal{C}, a}\right]_{u}$ the image of the Hessian in $\mathcal{O}\left(C_{\mathcal{C}, a}\right)_{u}$. We have

$$
\mathcal{R}_{u}:\left[\operatorname{Hess}_{\mathcal{C}, a}\right]_{u} \mapsto \mu_{u},
$$

where $\mu_{u}=\operatorname{dim}_{\mathbb{C}} \mathcal{O}\left(C_{\mathcal{C}, a}\right)_{u}$, the Milnor number, see Arnol'd et al. (1985). Define the bilinear form on $\mathcal{O}\left(C_{\mathcal{C}, a}\right)_{u}$,

$$
([f],[g])_{u}=\mathcal{R}_{u}([f][g]) .
$$

If $\mathcal{O}\left(C_{\mathcal{C}, a}\right)$ is finite-dimensional, we define the residue bilinear form $(,)_{C_{\mathcal{C}, a}}$ on $\mathcal{O}\left(C_{\mathcal{C}, a}\right)$ as

$$
(,)_{C_{\mathcal{C}, a}}=\oplus_{u \in C_{\mathcal{C}, a}}(,)_{u}
$$

This form is nondegenerate, see Arnol'd et al. (1985), and $([f][g],[h])_{C_{\mathcal{C}, a}}=$ $([f],[g][h])_{C_{\mathcal{C}, a}}$ for all $[f],[g],[h] \in \mathcal{O}\left(C_{\mathcal{C}, a}\right)$. In other words, the pair $\left(\mathcal{O}\left(C_{\mathcal{C}, a}\right)\right.$, $\left.(,)_{C_{\mathcal{C}, a}}\right)$ is a Frobenius algebra.

\subsection{Canonical Element}

A differential $k$-form $H \in \mathcal{A}^{k}(\mathcal{C})$ can be written as $H=f_{H} d t_{1} \wedge \cdots \wedge d t_{k}$, where $f_{H} \in \mathcal{O}(U(\mathcal{C}))$. Define a map F $: U(\mathcal{C}) \rightarrow \mathcal{F}^{k}(\mathcal{C})$ which sends $u \in U(\mathcal{C})$ to the 
element $\mathrm{F}(u) \in \mathcal{F}^{k}(\mathcal{C})$ such that $\langle H, \mathrm{~F}(u)\rangle=f_{H}(u)$ for any $H \in \mathcal{A}^{k}(\mathcal{C})$. The map $\mathrm{F}$ is called the specialization map, the vector $\mathrm{F}(u)$ is called the special vector at $u$, see Varchenko (2006). In the theory of quantum integrable systems special vectors are called the Bethe vectors.

Let $\left(F_{m}\right)_{m \in M}$ be a basis of $\mathcal{F}^{k}(\mathcal{C})$ and $\left(H^{m}\right)_{m \in M} \subset \mathcal{A}^{k}(\mathcal{C})$ the dual basis. We have $H^{m}=f_{H^{m}} d t_{1} \wedge \cdots \wedge d t_{k}$ for some $f_{H^{m}} \in \mathcal{O}(U(\mathcal{C}))$. The element

$$
E=\sum_{m \in M} f_{H^{m}} \otimes F_{m} \in \mathcal{O}(U(\mathcal{C})) \otimes \mathcal{F}^{k}(\mathcal{C})
$$

is called the canonical element. For $u \in U(\mathcal{C})$, we have

$$
\mathrm{F}(u)=\sum_{m \in M} f_{H^{m}}(u) F_{m}
$$

Let $[E]$ be the image of the canonical element in $\mathcal{O}\left(C_{\mathcal{C}, a}\right) \otimes \mathcal{F}^{k}(\mathcal{C})$.

Lemma 2.10 (Varchenko 2006, Lemma 2.6) We have $[E] \in \mathcal{O}\left(C_{\mathcal{C}, a}\right) \otimes \operatorname{Sing}_{a} \mathcal{F}^{k}(\mathcal{C})$.

Theorem 2.11 (Varchenko 2006) For $u \in U(\mathcal{C})$, we have

$$
S^{(a)}(\mathrm{F}(u), \mathrm{F}(u))=(-1)^{k} \operatorname{Hess}_{\mathcal{C}, a}(u) .
$$

Moreover, if $u^{1}, u^{2} \in U(\mathcal{C})$ are distinct isolated critical points of $\Phi_{\mathcal{C}, a}$, then the special singular vectors $\mathrm{F}\left(u^{1}\right), \mathrm{F}\left(u^{2}\right)$ are orthogonal,

$$
S^{(a)}\left(\mathrm{F}\left(u^{1}\right), \mathrm{F}\left(u^{2}\right)\right)=0,
$$

cf. Mukhin and Varchenko (2005), Varchenko (2011).

\subsection{Canonical Isomorphism}

Assume that the algebra $\mathcal{O}\left(C_{\mathcal{C}, a}\right)$ is finite-dimensional. Define the linear map

$$
\mathcal{E}: \mathcal{O}\left(C_{\mathcal{C}, a}\right) \rightarrow \operatorname{Sing} \mathcal{F}^{k}(\mathcal{C}), \quad[g] \mapsto([g],[E])_{C_{\mathcal{C}, a}}
$$

Theorem 2.12 (Varchenko 2011) If the weight $a \in\left(\mathbb{C}^{\times}\right)^{n}$ is unbalanced, then the map $\mathcal{E}$ is an isomorphism of vector spaces. The isomorphism $\mathcal{E}$ identifies the residue form on $\mathcal{O}\left(C_{\mathcal{C}, a}\right)$ and the contravariant form on $\operatorname{Sing} \mathcal{F}^{k}(\mathcal{C})$ multiplied by $(-1)^{k}$,

$$
(f, g)_{C_{\mathcal{C}, a}}=(-1)^{k} S^{(a)}(\mathcal{E}(f), \mathcal{E}(g)) \quad \text { for all } f, g \in \mathcal{O}\left(C_{\mathcal{C}, a}\right)
$$

The map $\mathcal{E}$ is called the canonical isomorphism. We provide a proof of Theorem 2.12 in Sect. 2.16.

Corollary 2.13 (Varchenko 2011) If the weight $a \in\left(\mathbb{C}^{\times}\right)^{n}$ is unbalanced, then the restriction of the contravariant form $S^{(a)}$ to the subspace $\operatorname{Sing} \mathcal{F}^{k}(\mathcal{C})$ is nondegenerate. 
On the restriction of $S^{(a)}$ to the subspace $\operatorname{Sing} \mathcal{F}^{k}(\mathcal{C})$ see also Falk and Varchenko (2012).

If all critical points are nondegenerate, then

$$
\mathcal{E}:[g] \mapsto \sum_{u \in C_{\mathcal{C}, a}} \sum_{m} \frac{g(u) f_{H^{m}}(u)}{\operatorname{Hess}_{\mathcal{C}, a}(u)} F_{m}=\sum_{u \in C_{\mathcal{C}, a}} \frac{g(u)}{\operatorname{Hess}_{\mathcal{C}, a}(u)} \mathrm{F}(u)
$$

see (2.10).

Remark. If the weight $a \in\left(\mathbb{C}^{\times}\right)^{n}$ is unbalanced, then the canonical isomorphism $\mathcal{E}$ induces a commutative associative algebra structure on $\operatorname{Sing}_{a} \mathcal{F}^{k}(\mathcal{C})$. Together with the contravariant form $\left.S^{(a)}\right|_{\operatorname{Sing}_{a} \mathcal{F}^{k}}$ it is a Frobenius algebra. The algebra of multiplication operators on $\operatorname{Sing}_{a} \mathcal{F}^{k}(\mathcal{C})$ is an analog of the Bethe algebra in the theory of quantum integrable models, see, for example, Mukhin et al. (2011), Varchenko (2011).

\subsection{Orthogonal Projection}

Lemma 2.14 It the weight $a \in\left(\mathbb{C}^{\times}\right)^{n}$ is unbalanced, then $d \mathcal{F}^{k-1}(\mathcal{C})=\operatorname{Sing}_{a}$ $\mathcal{F}^{k}(\mathcal{C})^{\perp}$, where $d \mathcal{F}^{k-1}(\mathcal{C}) \subset \mathcal{F}^{k}(\mathcal{C})$ is the image of the differential defined by (2.2) and $\operatorname{Sing}_{a} \mathcal{F}^{k}(\mathcal{C})^{\perp} \subset \mathcal{F}^{k}(\mathcal{C})$ is the orthogonal complement to $\operatorname{Sing}_{a} \mathcal{F}^{k}(\mathcal{C})$ with respect to $S^{(a)}$.

Proof We have $d \mathcal{F}^{k-1}(\mathcal{C}) \subset \operatorname{Sing}_{a} \mathcal{F}^{k}(\mathcal{C})^{\perp}$ by Lemma 2.2 and the definition of $\operatorname{Sing}_{a} \mathcal{F}^{k}(\mathcal{C})$. But $\operatorname{dim} d \mathcal{F}^{k-1}(\mathcal{C})=\operatorname{dim}_{\operatorname{Sing}_{a}} \mathcal{F}^{k}(\mathcal{C})^{\perp}$ by Theorem 2.1 and Corollary 2.5.

Corollary 2.15 It the weight $a \in\left(\mathbb{C}^{\times}\right)^{n}$ is unbalanced, the orthogonal projection $\pi^{\perp}: \mathcal{F}^{k}(\mathcal{C}) \rightarrow \operatorname{Sing}_{a} \mathcal{F}^{k}(\mathcal{C})$ establishes the isomorphism $H^{k}(\mathcal{F}(\mathcal{C}), d) \cong$ $\operatorname{Sing}_{a} \mathcal{F}^{k}(\mathcal{C})$.

Define the map

$$
\left[\mathcal{S}^{(a)}\right]: \mathcal{F}^{k}(\mathcal{C}) \rightarrow \mathcal{O}\left(C_{\mathcal{C}, a}\right), \quad F \mapsto \in[f],
$$

where $\mathcal{S}^{(a)}(F)=f d t_{1} \wedge \cdots \wedge d t_{k}$. Clearly, $\left[\mathcal{S}^{(a)}\right]\left(\operatorname{Sing}_{a} \mathcal{F}^{k}(\mathcal{C})^{\perp}\right)=\left[\mathcal{S}^{(a)}\right]\left(d \mathcal{F}^{k-1}(\mathcal{C})\right)$ $=0$, since $\omega^{(a)}=0$ on $C_{\mathcal{C}, a}$. In particular, $\left[\mathcal{S}^{(a)}\right]$ induces the map

$$
\left[\mathcal{S}^{(a)}\right]: H^{k}(\mathcal{F}(\mathcal{C}), d) \rightarrow \mathcal{O}\left(C_{\mathcal{C}, a}\right)
$$

Theorem 2.16 It the weight $a \in\left(\mathbb{C}^{\times}\right)^{n}$ is unbalanced, then the map

$$
\left.\left[\mathcal{S}^{(a)}\right]\right|_{\operatorname{Sing}_{a} \mathcal{F}^{k}(\mathcal{C})}: \operatorname{Sing}_{a} \mathcal{F}^{k}(\mathcal{C}) \rightarrow \mathcal{O}\left(C_{\mathcal{C}, a}\right)
$$

is an isomorphism of vector spaces and

$$
\left.\mathcal{E} \circ\left[\mathcal{S}^{(a)}\right]\right|_{\operatorname{Sing}_{a} \mathcal{F}^{k}(\mathcal{C})}=(-1)^{k} .
$$


Identity (2.22) was conjectured in Varchenko (2011). It was proved in Varchenko (2011) that the left-hand side in (2.22) is a nonzero scalar operator, if $\mathcal{C}$ is a generic arrangement.

Remark. The map $\left.\left[\mathcal{S}^{(a)}\right]\right|_{\operatorname{Sing}_{a} \mathcal{F}^{k}(\mathcal{C})}: \operatorname{Sing}_{a} \mathcal{F}^{k}(\mathcal{C}) \rightarrow \mathcal{O}\left(C_{\mathcal{C}, a}\right)$ is elementary. The map $\mathcal{E}: \mathcal{O}\left(C_{\mathcal{C}, a}\right) \rightarrow \operatorname{Sing}_{a} \mathcal{F}^{k}(\mathcal{C})$ is transcendental: it is given by a $k$-dimensional integral. Formula (2.22) says that the inverse map to the transcendental map is elementary.

Corollary 2.17 If the weight $a \in\left(\mathbb{C}^{\times}\right)^{n}$ is unbalanced, then the map $\left[\mathcal{S}^{(a)}\right]$ : $H^{k}(\mathcal{F}, d) \rightarrow \mathcal{O}\left(C_{\mathcal{C}, a}\right)$ is an isomorphism of vector spaces.

\subsection{Proof of Theorems 2.12 and 2.16}

First assume that the weight $a$ is generic and all critical points of $\Phi_{\mathcal{C}, a}$ are nondegenerate. Then the special vectors $(\mathrm{F}(u))_{u \in C_{\mathcal{C}, a}}$ form a basis of $\operatorname{Sing}_{a} \mathcal{F}^{k}(\mathcal{C})$ by Theorems 2.11, 2.7 and Corollary 2.5. (In the theory of quantum integrable systems this fact is called the completeness of the Bethe ansatz method, see Varchenko $(1995,2006)$.)

Theorem 2.11 and formula (2.10) applied to the basis $(\mathrm{F}(u))_{u \in C_{\mathcal{C}, a}}$ show that $S^{(a)}$ restricted to $\operatorname{Sing}_{a} \mathcal{F}^{k}(\mathcal{C})$ is nondegenerate and $\mathcal{E}: \mathcal{O}\left(C_{\mathcal{C}, a}\right) \rightarrow \operatorname{Sing}^{k}(\mathcal{C})$ is an isomorphism of vector spaces that identifies the residue form on $\mathcal{O}\left(C_{\mathcal{C}, a}\right)$ and the contravariant form on $\operatorname{Sing} \mathcal{F}^{k}(\mathcal{C})$ multiplied by $(-1)^{k}$. (More precisely, this follows from the following fact. Let $u \in C_{\mathcal{C}, a}$ and $g_{u} \in \mathcal{O}\left(C_{\mathcal{C}, a}\right)$ be the function which equals 1 at $u$ and equals 0 at other points of $C_{\mathcal{C}, a}$. Then $\mathcal{E}\left(g_{u}\right)=\mathrm{F}(u) / \operatorname{Hess}_{\mathcal{C}, a}(u)$.)

The orthogonal projection $\mathcal{F}^{k}(\mathcal{C}) \rightarrow \operatorname{Sing}_{a} \mathcal{F}^{k}(\mathcal{C})$ is defined by the formula

$$
F \mapsto \sum_{u \in C_{\mathcal{C}, a}} \frac{S^{(a)}(F, \mathrm{~F}(u))}{S^{(a)}(\mathrm{F}(u), \mathrm{F}(u))} \mathrm{F}(u)=(-1)^{k} \sum_{u \in C_{\mathcal{C}, a}} \frac{S^{(a)}(F, \mathrm{~F}(u))}{\operatorname{Hess}_{\mathcal{C}, a}(u)} \mathrm{F}(u) .
$$

Let $\mathcal{S}^{(a)}(F)=f d t_{1} \wedge \cdots \wedge d t_{k}$ and $u \in U(\mathcal{C})$, then $f(u)=S^{(a)}(F, \mathrm{~F}(u))$ by the definitions of $\mathrm{F}(u), \mathcal{S}^{(a)}, S^{(a)}$. Hence the map [ $\left.\mathcal{S}^{(a)}\right]$ defined in (2.19) sends $F$ to the element of $\mathcal{O}\left(C_{\mathcal{C}, a}\right)$ which equals $S^{(a)}(F, \mathrm{~F}(u))$ at every $u \in C_{\mathcal{C}, a}$. Applying formula (2.18) to this element we obtain

$$
\mathcal{E}_{0}\left[\mathcal{S}^{(a)}\right]: F \mapsto \sum_{u \in C_{\mathcal{C}, a}} \frac{S^{(a)}(F, \mathrm{~F}(u))}{\operatorname{Hess}_{\mathcal{C}, a}(u)} \mathrm{F}(u)
$$

These two formulas prove Theorem 2.16 if all critical points are nondegenerate.

Assume now that the weight $a$ is unbalanced. Then all critical points of $\Phi_{\mathcal{C}, a}$ are isolated and the sum of the corresponding Milnor numbers equals $|\chi(U(\mathcal{C}))|$. We deform the weight $a$ to make it generic and to make all critical points nondegenerate. Then $\operatorname{Sing}{ }_{a} \mathcal{F}(\mathcal{C})$ and $\left.S^{(a)}\right|_{\operatorname{Sing}_{a} \mathcal{F}(\mathcal{C})}$ continuously depend on the deformation as well as $\mathcal{O}\left(C_{\mathcal{C}, a}\right)$ and $(,)_{C_{\mathcal{C}, a}}$. The maps $\left.\left[\mathcal{S}^{(a)}\right]\right|_{\operatorname{Sing}_{a} \mathcal{F}(\mathcal{C})}$ and $\mathcal{E}$ also continuously depend on the deformation. This implies that for the initial unbalanced weight $a$, we have the 
identity $\left.\mathcal{E} \circ\left[\mathcal{S}^{(a)}\right]\right|_{\operatorname{Sing}_{a} \mathcal{F}^{k}(\mathcal{C})}=(-1)^{k}$ and the fact that $\mathcal{E}$ identifies the residue form on $\mathcal{O}\left(C_{\mathcal{C}, a}\right)$ and the contravariant form on $\operatorname{Sing} \mathcal{F}^{k}(\mathcal{C})$ multiplied by $(-1)^{k}$. This proves Theorems 2.16 and 2.12.

\subsection{Integral Structure on $\mathcal{O}\left(C_{\mathcal{C}, a}\right)$ and $\operatorname{Sing}_{a} \mathcal{F}^{k}(\mathcal{C})$}

If the weight $a$ is unbalanced, the formula $H^{p}(\mathcal{F}(\mathcal{C}), d)=H^{p}\left((\mathcal{F}(\mathcal{C}))_{\mathbb{Z}}, d_{\mathbb{Z}}\right) \otimes \mathbb{C}$ and the isomorphism $\left.\left[\mathcal{S}^{(a)}\right]\right|_{\operatorname{Sing}_{a} \mathcal{F}^{k}(\mathcal{C})}: H^{k}(\mathcal{F}, d) \rightarrow \mathcal{O}\left(C_{\mathcal{C}, a}\right)$ define an integral structure on $\mathcal{O}\left(C_{\mathcal{C}, a}\right)$. More precisely, for a $k$-flag of edges $X_{\alpha_{0}} \supset X_{\alpha_{1}} \supset \cdots \supset$ $X_{\alpha_{k}}$, let $\mathcal{S}^{(a)}\left(F_{\alpha_{0}, \ldots, \alpha_{k}}\right)=f_{\alpha_{0}, \ldots, \alpha_{k}} d t_{1} \wedge \cdots \wedge d t_{k}$. Denote by $w_{\alpha_{0}, \ldots, \alpha_{k}}$ the element $\left[f_{\alpha_{0}, \ldots, \alpha_{k}}\right] \in \mathcal{O}\left(C_{\mathcal{C}, a}\right)$.

Corollary 2.18 If the weight a is unbalanced, then the set of all elements $\left\{w_{\alpha_{0}, \ldots, \alpha_{k}}\right\}$, labeled by all $k$-flag of edges of $\mathcal{C}$, spans the vector space $\mathcal{O}\left(C_{\mathcal{C}, a}\right)$. All linear relations between the elements of the set are corollaries of the relations

$$
\begin{aligned}
& \sum_{X_{\beta}, X_{\alpha_{j-1}} \supset X_{\beta} \supset X_{\alpha_{j+1}}} w_{\alpha_{0}, \ldots, \alpha_{j-1}, \beta, \alpha_{j+1}, \ldots, \alpha_{p}=\alpha}=0, \\
& \sum_{X_{\beta}, X_{\alpha_{p}} \supset X_{\beta}} w_{\alpha_{0}, \ldots, \alpha_{p}, \beta}=0,
\end{aligned}
$$

cf. formulas (2.1), (2.2).

Similarly, for a $k$-flag of edges $X_{\alpha_{0}} \supset X_{\alpha_{1}} \supset \cdots \supset X_{\alpha_{k}}$, let $v_{\alpha_{0}, \ldots, \alpha_{k}}$ be the orthogonal projection of $F_{\alpha_{0}, \ldots, \alpha_{k}}$ to $\operatorname{Sing}_{a} \mathcal{F}^{k}(\mathcal{C})$.

Corollary 2.19 If the weight $a$ is unbalanced, then the set of all elements $\left\{v_{\alpha_{0}, \ldots, \alpha_{k}}\right\}$, labeled by all $k$-flag of edges of $\mathcal{C}$, spans the vector space $\operatorname{Sing}_{a} \mathcal{F}^{k}(\mathcal{C})$. All linear relations between the elements of the set are corollaries of the relations

$$
\begin{aligned}
& \sum_{X_{\beta}, X_{\alpha_{j-1}} \supset X_{\beta} \supset X_{\alpha_{j+1}}} v_{\alpha_{0}, \ldots, \alpha_{j-1}, \beta, \alpha_{j+1}, \ldots, \alpha_{p}=\alpha}=0, \\
& \sum_{X_{\beta}, X_{\alpha_{p}} \supset X_{\beta}} v_{\alpha_{0}, \ldots, \alpha_{p}, \beta}=0,
\end{aligned}
$$

cf. formulas (2.1), (2.2).

We have

$$
\left[\mathcal{S}^{(a)}\right]: v_{\alpha_{0}, \ldots, \alpha_{k}} \mapsto w_{\alpha_{0}, \ldots, \alpha_{k}}, \quad \mathcal{E}: w_{\alpha_{0}, \ldots, \alpha_{k}} \mapsto(-1)^{k} v_{\alpha_{0}, \ldots, \alpha_{k}}
$$

The elements $\left\{w_{\alpha_{0}, \ldots, \alpha_{k}}\right\} \subset \mathcal{O}\left(C_{\mathcal{C}, a}\right)$ and $\left\{v_{\alpha_{0}, \ldots, \alpha_{k}}\right\} \subset \operatorname{Sing}_{a} \mathcal{F}^{k}(\mathcal{C})$ will be called the marked elements. The relations (2.25), (2.26) will be called the marked relations.

Remark. An interesting problem is to express $1 \in \mathcal{O}\left(C_{\mathcal{C}, a}\right)$ as a linear combination of the marked elements $w_{\alpha_{0}, \ldots, \alpha_{k}}$, see Varchenko (2015), where such a formula is 
given for a generic arrangement. Notice, that if all points of the critical set $C_{\mathcal{C}, a}$ are nondegenerate, then

$$
\mathcal{E}(1)=\sum_{u \in C_{\mathcal{C}, a}} \mathrm{~F}(u) / \operatorname{Hess}_{\mathcal{C}, a}(u)
$$

see Mukhin et al. (2011), where such sums were studied.

\subsection{Skew-Commutative Versus Commutative}

If $a$ is unbalanced, then

$$
H^{k}\left(\mathcal{A}(\mathcal{C}), d^{(a)}\right) \cong H^{k}(\mathcal{F}(\mathcal{C}), d) \cong \mathcal{O}\left(C_{\mathcal{C}, a}\right)
$$

as vector spaces. The first space is a cohomology space of a skew-commutative graded algebra $\mathcal{A}(\mathcal{C})$. The last space is the vector space of a commutative algebra. Isomorphisms (2.28) identify these skew-commutative and commutative objects. It is interesting to identify the multiplication operators on the last space with suitable operators on the first two spaces. It turns out that those operators appear in the associated Gauss-Manin (hypergeometric) differential equations, see Sect. 3.7 and Mukhin et al. (2011), Varchenko (2006), Varchenko (2011).

Another identification of skew-commutative and commutative objects of an arrangement see in Gelfand and Varchenko (1987), Proudfoot (2006).

\subsection{Combinatorial Connection}

Consider a deformation $\mathcal{C}(s)$ of the arrangement $\mathcal{C}$, which preserves the combinatorics of $\mathcal{C}$. Assume that the edges of $\mathcal{C}(s)$ can be identified with the edges of $\mathcal{C}$ so that the elements in formula (2.1) and the differential in formula (2.2) do not depend on $s$. Assume that the deformed arrangement $\mathcal{C}(s)$ has a deformed weight $a(s)$, which remains unbalanced. Then for every $s$, the elements $\left\{w_{\alpha_{0}, \ldots, \alpha_{k}}(s)\right\}$ span $\mathcal{O}\left(C_{\mathcal{C}(s), a(s)}\right)$ as a vector space with linear relations (2.25) not depending on $s$. This allows us to identify all the vector spaces $\mathcal{O}\left(C_{\mathcal{C}(s), a(s)}\right)$. In particular, if an element $w(s) \in \mathcal{O}\left(C_{\mathcal{C}(s), a(s)}\right)$ is given, then the derivative $\frac{d w}{d s}$ is well-defined. This construction is called the combinatorial connection on the family of algebras $\mathcal{O}\left(C_{\mathcal{C}}(s), a(s)\right.$, see Varchenko (2015). All the elements $\left\{w_{\alpha_{0}, \ldots, \alpha_{k}}(s)\right\}$ are flat sections of the combinatorial connection.

Similarly we can define the combinatorial connection on the family of vector spaces $\operatorname{Sing}_{a(s)} \mathcal{F}^{k}(\mathcal{C}(s))$.

\subsection{Arrangement with Normal Crossings}

An essential arrangement $\mathcal{C}$ is with normal crossings, if exactly $k$ hyperplanes meet at every vertex of $\mathcal{C}$. Assume that $\mathcal{C}$ is an essential arrangement with normal crossings. A subset $\left\{j_{1}, \ldots, j_{p}\right\} \subset J$ is called independent if the hyperplanes $H_{j_{1}}, \ldots, H_{j_{p}}$ intersect transversally. 
A basis of $\mathcal{A}^{p}(\mathcal{C})$ is formed by $\left(H_{j_{1}}, \ldots, H_{j_{p}}\right)$, where $\left\{j_{1}<\cdots<j_{p}\right\}$ are independent ordered $p$-element subsets of $J$. The dual basis of $\mathcal{F}^{p}(\mathcal{C})$ is formed by the corresponding vectors $F\left(H_{j_{1}}, \ldots, H_{j_{p}}\right)$. These bases of $\mathcal{A}^{p}(\mathcal{C})$ and $\mathcal{F}^{p}(\mathcal{C})$ are called standard. We have

$$
F\left(H_{j_{1}}, \ldots, H_{j_{p}}\right)=(-1)^{|\sigma|} F\left(H_{j_{\sigma(1)}}, \ldots, H_{j_{\sigma(p)}}\right), \quad \text { for } \quad \sigma \in \Sigma_{p}
$$

For an independent subset $\left\{j_{1}, \ldots, j_{p}\right\}$, we have $S^{(a)}\left(F\left(H_{j_{1}}, \ldots, H_{j_{p}}\right), F\left(H_{j_{1}}, \ldots\right.\right.$, $\left.\left.H_{j_{p}}\right)\right)=a_{j_{1}} \cdots a_{j_{p}}$ and $S^{(a)}\left(F\left(H_{j_{1}}, \ldots, H_{j_{p}}\right), F\left(H_{i_{1}}, \ldots, H_{i_{k}}\right)\right)=0$ for distinct elements of the standard basis. If $a$ is unbalanced, then the marked elements in $\mathcal{O}\left(C_{\mathcal{C}, a}\right)$ are

$$
w_{i_{1}, \ldots, i_{k}}=d_{i_{1}, \ldots, i_{k}} \frac{a_{i_{1}}}{\left[f_{i_{1}}\right]} \ldots \frac{a_{i_{k}}}{\left[f_{i_{k}}\right]},
$$

where $\left\{i_{1}, \ldots, i_{k}\right\}$ runs through the set of all independent $k$-element subsets of $J$. We have

$$
w_{i_{\sigma(1)}, \ldots, i_{\sigma(k)}}=(-1)^{\sigma} w_{i_{1}, \ldots, i_{k}}, \quad \text { for } \sigma \in \Sigma_{k}
$$

We put $w_{i_{1}, \ldots, i_{k}}=0$ if the set $\left\{i_{1}, \ldots, i_{k}\right\}$ is dependent. The marked relations are labeled by independent subsets $\left\{i_{2}, \ldots, i_{k}\right\}$ and have the form

$$
\sum_{j \in J} w_{j, i_{2}, \ldots, i_{k}}=0
$$

The marked elements $v_{i_{1}, \ldots, i_{k}}$ in $\operatorname{Sing}_{a} \mathcal{F}^{k}(\mathcal{C})$ are orthogonal projections to $\operatorname{Sing}_{a} \mathcal{F}^{k}(\mathcal{C})$ of the elements $F\left(H_{i_{1}}, \ldots, H_{i_{k}}\right)$ with the skew-symmetry property

$$
v_{i_{\sigma(1)}, \ldots, i_{\sigma(k)}}=(-1)^{\sigma} v_{i_{1}, \ldots, i_{k}}, \quad \text { for } \sigma \in \Sigma_{k} \text {. }
$$

and the marked relations

$$
\sum_{j \in J} v_{j, i_{2}, \ldots, i_{k}}=0
$$

labeled by independent subsets $\left\{i_{2}, \ldots, i_{k}\right\}$.

\section{Family of Parallelly Transported Hyperplanes}

\subsection{Arrangement in $\mathbb{C}^{n} \times \mathbb{C}^{k}$}

Recall that $J=\{1, \ldots, n\}$. Consider $\mathbb{C}^{k}$ with coordinates $t_{1}, \ldots, t_{k}, \mathbb{C}^{n}$ with coordinates $z_{1}, \ldots, z_{n}$, the projection $\pi: \mathbb{C}^{n} \times \mathbb{C}^{k} \rightarrow \mathbb{C}^{n}$. Fix $n$ nonzero linear functions on $\mathbb{C}^{k}, g_{j}=b_{j}^{1} t_{1}+\cdots+b_{j}^{k} t_{k}, j \in J$, where $b_{j}^{i} \in \mathbb{C}$. We assume that the functions $g_{j}, j \in J$, span the dual space $\left(\mathbb{C}^{k}\right)^{*}$. 
Define $n$ linear functions on $\mathbb{C}^{n} \times \mathbb{C}^{k}, f_{j}=g_{j}+z_{j}=b_{j}^{1} t_{1}+\cdots+b_{j}^{k} t_{k}+z_{j}$, $j \in J$. We consider in $\mathbb{C}^{n} \times \mathbb{C}^{k}$ the arrangement of hyperplanes $\mathcal{C}=\left\{H_{j}\right\}_{j \in J}$, where $H_{j}$ is the zero set of $f_{j}$, and denote by $U(\mathcal{C})=\mathbb{C}^{n} \times \mathbb{C}^{k}-\cup_{j \in J} H_{j}$ the complement.

Lemma 3.1 For any linear relation $\sum_{j=1}^{n} \beta_{j} g_{j}=0$ we have the relation

$$
\sum_{j=1}^{n} \beta_{j}\left(z_{j}-f_{j}\right)=0
$$

For every $x=\left(x_{1}, \ldots, x_{n}\right) \in \mathbb{C}^{n}$, the arrangement $\mathcal{C}$ induces an arrangement $\mathcal{C}(x)$ in the fiber $\pi^{-1}(x)$. We identify every fiber with $\mathbb{C}^{k}$. Then $\mathcal{C}(x)$ consists of hyperplanes $\left\{H_{j}(x)\right\}_{j \in J}$, defined in $\mathbb{C}^{k}$ by the equations $g_{j}+x_{j}=0$. Thus $\{\mathcal{C}(x)\}_{x \in \mathbb{C}^{n}}$ is a family of arrangements in $\mathbb{C}^{k}$, whose hyperplanes are transported parallelly as $x$ changes. We denote by $U(\mathcal{C}(x))=\mathbb{C}^{k}-\cup_{j \in J} H_{j}(x)$ the complement.

For almost all points $x \in \mathbb{C}^{n}$, the arrangement $\mathcal{C}(x)$ is with normal crossings. Such points form the complement in $\mathbb{C}^{n}$ to the union of suitable hyperplanes called the discriminant.

\subsection{Discriminant}

The collection $\left(g_{j}\right)_{j \in J}$ induces a matroid structure on $J$. A subset $C=\left\{i_{1}, \ldots, i_{r}\right\} \subset$ $J$ is a circuit if $\left(g_{i}\right)_{i \in C}$ are linearly dependent but any proper subset of $C$ gives linearly independent $g_{i}$ 's. Denote by $\mathfrak{C}$ the set of all circuits in $J$.

For a circuit $C=\left\{i_{1}, \ldots, i_{r}\right\}$, let $\left(\lambda_{i}^{C}\right)_{i \in C}$ be a nonzero collection of complex numbers such that $\sum_{i \in C} \lambda_{i}^{C} g_{i}=0$. Such a collection is unique up to multiplication by a nonzero number. For every circuit $C$ we fix such a collection and denote $f_{C}=$ $\sum_{i \in C} \lambda_{i}^{C} z_{i}$. The equation $f_{C}=0$ defines a hyperplane $H_{C}$ in $\mathbb{C}^{n}$. It is convenient to assume that $\lambda_{i}^{C}=0$ for $i \in J-C$ and write $f_{C}=\sum_{i \in J} \lambda_{i}^{C} z_{i}$.

Lemma 3.2 Any linear relation $\sum_{j \in J} c_{j} g_{j}=0$ is a linear combination of relations $\sum_{i \in J} \lambda_{i}^{C} g_{i}=0$ associated with circuits $C \in \mathfrak{C}$.

For any $x \in \mathbb{C}^{n}$, the hyperplanes $\left\{H_{i}(x)\right\}_{i \in C}$ in $\mathbb{C}^{k}$ have nonempty intersection if and only if $x \in H_{C}$. If $x \in H_{C}$, then the intersection has codimension $r-1$ in $\mathbb{C}^{k}$. The union $\Delta=\cup_{C \in \mathfrak{C}} H_{C}$ is called the discriminant. The arrangement $\mathcal{C}(x)$ in $\mathbb{C}^{k}$ has normal crossings if and only if $x \in \mathbb{C}^{n}-\Delta$, see Varchenko (2011).

On the discriminant see also Bayer and Brandt (1997).

\subsection{Combinatorial Connection}

For any $x^{1}, x^{2} \in \mathbb{C}^{n}-\Delta$, the spaces $\mathcal{F}^{p}\left(\mathcal{C}\left(x^{1}\right)\right), \mathcal{F}^{p}\left(\mathcal{C}\left(x^{2}\right)\right)$ are canonically identified if a vector $F\left(H_{j_{1}}\left(x^{1}\right), \ldots, H_{j_{p}}\left(x^{1}\right)\right)$ of the first space is identified with the vector $F\left(H_{j_{1}}\left(x^{2}\right), \ldots, H_{j_{p}}\left(x^{2}\right)\right)$ of the second. In other words, we identify the standard bases of these spaces. 
Assume that a weight $a \in\left(\mathbb{C}^{\times}\right)^{n}$ is given. Then each arrangement $\mathcal{C}(x)$ is weighted. The identification of spaces $\mathcal{F}^{p}\left(\mathcal{C}\left(x^{1}\right)\right), \mathcal{F}^{p}\left(\mathcal{C}\left(x^{2}\right)\right)$ for $x^{1}, x^{2} \in \mathbb{C}^{n}-\Delta$ identifies the corresponding subspaces $\operatorname{Sing}_{a} \mathcal{F}^{k}\left(\mathcal{C}\left(x^{1}\right)\right), \operatorname{Sing}_{a} \mathcal{F}^{k}\left(\mathcal{C}\left(x^{2}\right)\right)$ and contravariant forms.

Assume that the weighted arrangement $(\mathcal{C}(x), a)$ is unbalanced for some $x \in$ $\mathbb{C}^{n}-\Delta$, then $(\mathcal{C}(x), a)$ is unbalanced for all $x \in \mathbb{C}^{n}-\Delta$. The identification of $\operatorname{Sing}_{a} \mathcal{F}^{k}\left(\mathcal{C}\left(x^{1}\right)\right)$ and $\operatorname{Sing}_{a} \mathcal{F}^{k}\left(\mathcal{C}\left(x^{2}\right)\right)$ also identifies the marked elements $v_{j_{1}, \ldots, j_{k}}\left(x^{1}\right)$ and $v_{j_{1}, \ldots, j_{k}}\left(x^{1}\right)$, see Sect. 2.20. For $x \in \mathbb{C}^{n}-\Delta$, denote $V=\mathcal{F}^{k}(\mathcal{C}(x))$, $\operatorname{Sing}_{a} V=\operatorname{Sing}_{a} \mathcal{F}^{k}(\mathcal{C}(x)), v_{j_{1}, \ldots, j_{k}}=v_{j_{1}, \ldots, j_{k}}(x)$. The triple $\left(V, \operatorname{Sing}_{a} V, S^{(a)}\right)$, with marked elements $v_{j_{1}, \ldots, j_{k}}$, does not depend on $x$ under the identification.

As a result of this reasoning we obtain the canonically trivialized vector bundle

$$
\sqcup_{x \in \mathbb{C}^{n}-\Delta} \mathcal{F}^{k}(\mathcal{C}(x)) \rightarrow \mathbb{C}^{n}-\Delta
$$

with the canonically trivialized subbundle $\sqcup_{x \in \mathbb{C}^{n}-\Delta} \operatorname{Sing}_{a} \mathcal{F}^{k}(\mathcal{C}(x)) \rightarrow \mathbb{C}^{n}-\Delta$ and the constant contravariant form on the fibers. This trivialization identifies the bundle in (3.2) with the bundle $\left(\mathbb{C}^{n}-\Delta\right) \times V \rightarrow \mathbb{C}^{n}-\Delta$ and the subbundle $\sqcup_{x \in \mathbb{C}^{n}-\Delta} \operatorname{Sing}_{a} \mathcal{F}^{k}(\mathcal{C}(x)) \rightarrow \mathbb{C}^{n}-\Delta$ with the subbbundle

$$
\left(\mathbb{C}^{n}-\Delta\right) \times\left(\operatorname{Sing}_{a} V\right) \rightarrow \mathbb{C}^{n}-\Delta .
$$

The bundle in (3.3) will be called the combinatorial bundle, the flat connection on it will be called combinatorial, see Varchenko (2011, 2015), cf. Sect. 2.19.

\subsection{Operators $K_{j} \in \mathcal{O}\left(\mathbb{C}^{n}-\Delta\right) \otimes($ End $V), j \in J$}

For a circuit $C=\left\{i_{1}, \ldots, i_{r}\right\} \subset J$, we define the linear operator $L_{C}: V \rightarrow V$ as follows. Let $C_{m}=C-\left\{i_{m}\right\}$. Let $F\left(H_{j_{1}}, \ldots, H_{j_{k}}\right)$ be an element of the standard basis. We set $L_{C}: F\left(H_{j_{1}}, \ldots, H_{j_{k}}\right) \mapsto 0$ if $\left|\left\{j_{1}, \ldots, j_{k}\right\} \cap C\right|<$ $r-1$. If $\left\{j_{1}, \ldots, j_{k}\right\} \cap C=C_{m}$, then by (2.29) we have $F\left(H_{j_{1}}, \ldots, H_{j_{k}}\right)=$ $\pm F\left(H_{i_{1}}, H_{i_{2}}, \ldots, \widehat{H_{i_{m}}}, \ldots, H_{i_{r-1}}, H_{i_{r}}, H_{s_{1}}, \ldots, H_{s_{k-r+1}}\right)$ with $\left\{s_{1}, \ldots, s_{k-r+1}\right\}=$ $\left\{j_{1}, \ldots, j_{k}\right\}-C_{m}$. We set

$$
\begin{aligned}
L_{C} & : F\left(H_{i_{1}}, \ldots, \widehat{H_{i_{m}}}, \ldots, H_{i_{r}}, H_{s_{1}}, \ldots, H_{s_{k-r+1}}\right) \\
& \mapsto(-1)^{m} \sum_{l=1}^{r}(-1)^{l} a_{i_{l}} F\left(H_{i_{1}}, \ldots, \widehat{H_{i_{l}}}, \ldots, H_{i_{r}}, H_{s_{1}}, \ldots, H_{s_{k-r+1}}\right) .
\end{aligned}
$$

Consider on $\mathbb{C}^{n} \times \mathbb{C}^{k}$ the logarithmic 1-forms $\omega_{C}=\frac{d f_{C}}{f_{C}}, C \in \mathfrak{C}$. Recall $f_{C}=$ $\sum_{j \in J} \lambda_{j}^{C} z_{j}$. We set

$$
K_{j}=\sum_{C \in \mathfrak{C}} \frac{\lambda_{j}^{C}}{f_{C}} L_{C} \in \mathcal{O}\left(\mathbb{C}^{n}-\Delta\right) \otimes(\text { End } V)
$$

We have

$$
\sum_{C \in \mathfrak{C}} \omega_{C} \otimes L_{C}=\sum_{j \in J} d z_{j} \otimes K_{j} .
$$


Theorem 3.3 (Varchenko 2011) For any $j \in J$ and $x \in \mathbb{C}^{n}-\Delta$, the operator $K_{j}(x)$ preserves the subspace $\operatorname{Sing}_{a} V \subset V$ and is a symmetric operator, $S^{(a)}\left(K_{j}(x) v, w\right)=$ $S^{(a)}\left(v, K_{j}(x) w\right)$ for all $v, w \in V$.

\subsection{Corollary of Theorem 3.3}

We obtain formulas for the action of $K_{j}$ on the marked elements $v_{j_{1}, \ldots, j_{k}} \in \operatorname{Sing}_{a} V$ from formulas for the action of $L_{C}$.

Let $C=\left\{i_{1}, \ldots, i_{r}\right\}$ be a circuit and $v_{j_{1}, \ldots, j_{k}} \in \operatorname{Sing}_{a} V$ a marked element. If $\left|\left\{j_{1}, \ldots, j_{k}\right\} \cap C\right|<r-1$, then $L_{C}\left(v_{j_{1}, \ldots, j_{k}}\right)=0$. If $\left\{j_{1}, \ldots, j_{k}\right\} \cap C=C_{m}$, then by (2.33) we have $v_{j_{1}, \ldots, j_{k}}= \pm v_{i_{1}, i_{2}, \ldots, \widehat{i_{m}}, \ldots, i_{r-1}, i_{r}, s_{1}, \ldots, s_{k-r+1}}$ with $\left\{s_{1}, \ldots, s_{k-r+1}\right\}=$ $\left\{j_{1}, \ldots, j_{k}\right\}-C_{m}$. We have

$L_{C}: v_{i_{1}, i_{2}, \ldots, \hat{i_{m}}, \ldots, i_{r-1}, i_{r}, s_{1}, \ldots, s_{k-r+1}} \mapsto(-1)^{m} \sum_{l=1}^{r}(-1)^{l} a_{i_{l}} v_{i_{1}, \ldots, \widehat{i_{l}}, \ldots, i_{r}, s_{1}, \ldots, s_{k-r+1}}$

\subsection{Gauss-Manin Connection on $\left(\mathbb{C}^{n}-\Delta\right) \times\left(\operatorname{Sing}_{a} V\right) \rightarrow \mathbb{C}^{n}-\Delta$}

The master function of $(\mathcal{C}, a)$ is $\Phi_{\mathcal{C}, a}=\sum_{j \in J} a_{j} \log f_{j}$, a multivalued function on $U(\mathcal{C})$. Let $\kappa \in \mathbb{C}^{\times}$. The function $e^{\Phi_{\mathcal{C}, a} / \kappa}$ defines a rank one local system $\mathcal{L}_{\kappa}$ on $U(\mathcal{C})$ whose horizontal sections over open subsets of $U(\mathcal{C})$ are univalued branches of $e^{\Phi_{\mathcal{C}, a} / \kappa}$ multiplied by complex numbers, see, for example, Schechtman and Varchenko (1991), Varchenko (1995). The vector bundle

$$
\sqcup_{x \in \mathbb{C}^{n}-\Delta} H_{k}\left(U(\mathcal{C}(x)),\left.\mathcal{L}_{\kappa}\right|_{U(\mathcal{C}(x))}\right) \rightarrow \mathbb{C}^{n}-\Delta
$$

is called the homology bundle. The homology bundle has a canonical flat Gauss-Manin connection.

For a fixed $x$, choose any $\gamma \in H_{k}\left(U(\mathcal{C}(x)),\left.\mathcal{L}_{\kappa}\right|_{U(\mathcal{C}(x))}\right)$. The linear map

$$
\{\gamma\}: \mathcal{A}^{k}(\mathcal{C}(x)) \rightarrow \mathbb{C}, \quad \omega \mapsto \int_{\gamma} e^{\Phi_{\mathcal{C}, a} / \kappa} \omega,
$$

is an element of $\operatorname{Sing} \mathcal{F}^{k}(\mathcal{C}(x))$ by Stokes' theorem. It is known that for generic $\kappa$ any element of $\operatorname{Sing} \mathcal{F}^{k}(\mathcal{C}(x))$ corresponds to a certain $\gamma$ and in that case this construction gives the integration isomorphism

$$
H_{k}\left(U(\mathcal{C}(x)),\left.\mathcal{L}_{\kappa}\right|_{U(\mathcal{C}(x))}\right) \rightarrow \operatorname{Sing}_{a} \mathcal{F}^{k}(\mathcal{C}(x)),
$$

see Schechtman and Varchenko (1991). The precise values of $\kappa$, such that (3.10) is an isomorphism, can be deduced from the determinant formula in Varchenko (1989).

For generic $\kappa$ the fiber isomorphisms (3.10) define an isomorphism of the homology bundle and the combinatorial bundle (3.3). The Gauss-Manin connection induces a 
connection on the combinatorial bundle. That connection on the combinatorial bundle will also be called the Gauss-Manin connection.

Thus, there are two connections on the combinatorial bundle: the combinatorial connection and the Gauss-Manin connection depending on $\kappa$. In this situation we consider the differential equations for flat sections of the Gauss-Manin connection with respect to the combinatorially flat standard basis. Namely, let $\gamma(x) \in$ $H_{k}\left(U(\mathcal{C}(x)),\left.\mathcal{L}_{\kappa}\right|_{U(\mathcal{C}(x))}\right)$ be a flat section of the Gauss-Manin connection. Let us write the corresponding section $I_{\gamma}(x)$ of the bundle $\left(\mathbb{C}^{n}-\Delta\right) \times \operatorname{Sing}_{a} V \rightarrow \mathbb{C}^{n}-\Delta$ in the combinatorially flat standard basis,

$$
\begin{aligned}
I_{\gamma}(x) & =\sum_{\substack{\text { independent } \\
\left\{j_{1}<\cdots<j_{k}\right\} \subset J}} I_{\gamma}^{j_{1}, \ldots, j_{k}}(x) F\left(H_{j_{1}}, \ldots, H_{j_{k}}\right), \\
I_{\gamma}^{j_{1}, \ldots, j_{k}}(x) & =\int_{\gamma(x)} e^{\Phi_{\mathcal{C}, a} / \kappa} \omega_{j_{1}} \wedge \cdots \wedge \omega_{j_{k}} .
\end{aligned}
$$

By Theorem 3.3, we can also write

$$
I_{\gamma}(x)=\sum_{\substack{\text { independent } \\\left\{j_{1}<\cdots<j_{k}\right\} \subset J}} I_{\gamma}^{j_{1}, \ldots, j_{k}}(x) v_{j_{1}, \ldots, j_{k}} .
$$

For $I=\sum I^{j_{1}, \ldots, j_{k}} v_{j_{1}, \ldots, j_{k}}$ and $j \in J$, we denote $\frac{\partial I}{\partial z_{j}}=\sum \frac{\partial I^{j_{1}, \ldots, j_{k}}}{\partial z_{j}} v_{j_{1}, \ldots, j_{k}}$. This formula defines the combinatorial connection on the combinatorial bundle.

Theorem 3.4 (Varchenko 1995, 2011) The section $I_{\gamma}$ satisfies the differential equations

$$
\kappa \frac{\partial I}{\partial z_{j}}(x)=K_{j}(x) I(x), \quad j \in J,
$$

where $K_{j}(x)$ are the linear operators defined in (3.5).

On the Gauss-Manin connection and these differential equations see also Cohen and Orlik (2006).

\subsection{Critical Set}

Denote by $C_{\mathcal{C}, a}$ the critical set of $\Phi_{\mathcal{C}, a}$ in the $\mathbb{C}^{k}$-direction,

$$
C_{\mathcal{C}, a}=\left\{(x, u) \in U(\mathcal{C}) \subset \mathbb{C}^{n} \times \mathbb{C}^{k} \mid \frac{\partial \Phi_{\mathcal{C}, a}}{\partial t_{i}}(x, u)=0 \quad \text { for } \quad i=1, \ldots, k\right\} .
$$

Lemma 3.5 If $C_{\mathcal{C}, a}$ is nonempty, then it is a smooth n-dimensional subvariety of $U(\mathcal{C})$. 
Proof For $j_{1}, \ldots, j_{k} \in J$, we have

$$
\operatorname{det}_{i, l=1}^{k}\left(\frac{\partial^{2} \Phi_{\mathcal{C}, a}}{\partial z_{j_{l}} \partial t_{i}}\right)=(-1)^{k} \operatorname{det}_{i, l=1}^{k}\left(b_{j_{l}}^{i}\right) \prod_{l=1}^{k} \frac{a_{j_{l}}}{f_{j_{l}}^{2}} .
$$

Since $\left(g_{j}\right)_{j \in J}$ span $\left(\mathbb{C}^{k}\right)^{*}$, there exists $j_{1}, \ldots, j_{k} \in J$ such that $\operatorname{det}_{i, l=1}^{k}\left(b_{j_{l}}^{i}\right) \neq 0$.

Lemma 3.6 If $a \in\left(\mathbb{C}^{\times}\right)^{n}$ is generic, then

(i) every fiber of the projection $\left.\pi\right|_{C_{\mathcal{C}, a}}: C_{\mathcal{C}, a} \rightarrow \mathbb{C}^{n}$ is finite;

(ii) for any $x \in \mathbb{C}^{n}$, the number of points of $C_{\mathcal{C}, a}$ in the fiber over $x$, counted with their Milnor numbers, equals $|\chi(U(\mathcal{C}(x)))|$;

(iii) for generic $x \in \mathbb{C}^{n}$, each of the points of $C_{\mathcal{C}, a}$ in the fiber over $x$ is nondegenerate.

Proof The lemma follows from Theorem 2.7 and Lemma 2.8.

Let $\mathcal{O}\left(C_{\mathcal{C}, a}\right)$ be the algebra of regular functions on $C_{\mathcal{C}, a}$ and $\mathcal{O}\left(C_{\mathcal{C}(x), a}\right)$ the algebra of regular functions on $C_{\mathcal{C}(x), a}=C_{\mathcal{C}, a} \cap \pi^{-1}(x)$. Namely, for $x=\left(x_{1}, \ldots, x_{n}\right) \in \mathbb{C}^{n}$, let $I_{\mathcal{C}(x), a}$ be the ideal in $\mathcal{O}(U(\mathcal{C}(x)))$ generated by $\frac{\partial \Phi_{\mathcal{C}, a}}{\partial t_{i}}, i=1, \ldots, k$. We set

$$
\mathcal{O}\left(C_{\mathcal{C}(x), a}\right)=\mathcal{O}(U(\mathcal{C}(x))) / I_{\mathcal{C}(x), a}
$$

Assume that the weight $a$ is such that the pair $(\mathcal{C}(x), a)$ is unbalanced for some $x \in \mathbb{C}^{n}-\Delta$. Then $\operatorname{dim} \mathcal{O}\left(C_{\mathcal{C}(x), a}\right)=|\chi(U(\mathcal{C}(x)))|$ for every $x \in \mathbb{C}^{n}-\Delta$ and we obtain the vector bundle of algebras

$$
\sqcup_{x \in \mathbb{C}^{n}-\Delta} \mathcal{O}\left(C_{\mathcal{C}(x), a}\right) \rightarrow \mathbb{C}^{n}-\Delta
$$

For $x \in \mathbb{C}^{n}-\Delta$, consider the canonical element $E(x)$ of the arrangement $\mathcal{C}(x)$ and its image $[E(x)]$ in $\mathcal{O}\left(C_{\mathcal{C}(x), a}\right) \otimes \operatorname{Sing}_{a} V$, see Lemma 2.10. Recall the canonical isomorphism (2.16),

$$
\mathcal{E}(x): \mathcal{O}\left(C_{\mathcal{C}(x), a}\right) \rightarrow \operatorname{Sing}_{a} V
$$

This fiber isomorphism establishes an isomorphism $\mathcal{E}$ of the bundles (3.16) and (3.3). The isomorphism $\mathcal{E}$ and the combinatorial and Gauss-Manin connections on the bundle (3.3) induce two connections on the bundle (3.16) which will also be called the combinatorial and Gauss-Manin connections, respectively.

Theorem 3.7 (Varchenko 2011) If the pair $(\mathcal{C}(x), a)$ is unbalanced for $x \in \mathbb{C}^{n}-\Delta$, then for all $j \in J$, we have

$$
\mathcal{E}(x) \circ\left[\frac{a_{j}}{f_{j}}\right] *_{x}=K_{j}(x) \circ \mathcal{E}(x),
$$

where $\left[\frac{a_{j}}{f_{j}}\right] *_{x}$ is the operator of multiplication by $\left[\frac{a_{j}}{f_{j}}\right]$ in $\mathcal{O}\left(C_{\mathcal{C}(x), a}\right)$ and $K_{j}(x)$ : $\operatorname{Sing}_{a} V \rightarrow \operatorname{Sing}_{a} V$ is the operator defined in (3.5). 
Remark. Recall that $a_{j} / f_{j}=\partial \Phi_{\mathcal{C}, a} / \partial z_{j}$ and the elements $\left[a_{j} / f_{j}\right], j \in J$, generate the algebra $\mathcal{O}\left(C_{\mathcal{C}(x), a}\right)$. Theorem (3.7) says that under the isomorphism $\mathcal{E}(x)$ the operators of multiplication $\left[a_{j} / f_{j}\right] *_{x}$ in $\mathcal{O}\left(C_{\mathcal{C}(x), a}\right)$ are identified with the operators $K_{j}(x)$ in the Gauss-Manin differential equations (3.13), cf. Sect. 2.18. The correspondence of Theorem 3.7 defines a commutative algebra structure on $\operatorname{Sing}_{a} V$, the structure depending on $x$. The multiplication in this commutative algebra is generated by the operators $K_{j}(x), j \in J$. The correspondence of Theorem 3.7 also defines the GaussManin differential equations on the bundle of algebras in terms of the multiplication in the fiber algebras.

Notice that the relations between the operators $K_{j}(x)$ coincide with the relations among the elements $\left[a_{j} / f_{j}\right]$ in $C_{\mathcal{C}}(x), a$.

\subsection{Formulas for Multiplication}

For $x \in \mathbb{C}^{n}-\Delta$ and a circuit $C=\left\{i_{1}, \ldots, i_{r}\right\} \subset J$, we define the linear operator $L_{C}: \mathcal{O}\left(C_{\mathcal{C}(x), a}\right) \rightarrow \mathcal{O}\left(C_{\mathcal{C}(x), a}\right)$ as follows. Let $w_{j_{1}, \ldots, j_{k}} \in \mathcal{O}\left(C_{\mathcal{C}(x), a}\right)$ be a marked element. If $\left|\left\{j_{1}, \ldots, j_{k}\right\} \cap C\right|<r-1$, then we set $L_{C}\left(w_{j_{1}, \ldots, j_{k}}\right)=0$. If $\left\{j_{1}, \ldots, j_{k}\right\} \cap$ $C=C_{m}$, then by (2.31) we have $w_{j_{1}, \ldots, j_{k}}= \pm w_{i_{1}, i_{2}, \ldots, \widehat{i_{m}}, \ldots, i_{r-1}, i_{r}, s_{1}, \ldots, s_{k-r+1}}$ with $\left\{s_{1}, \ldots, s_{k-r+1}\right\}=\left\{j_{1}, \ldots, j_{k}\right\}-C_{m}$. We set

$$
L_{C}\left(w_{i_{1}, i_{2}, \ldots, \widehat{i_{m}}, \ldots, i_{r-1}, i_{r}, s_{1}, \ldots, s_{k-r+1}}\right)=(-1)^{m} \sum_{l=1}^{r}(-1)^{l} a_{i_{l}} w_{i_{1}, \ldots, \widehat{i_{l}}, \ldots, i_{r}, s_{1}, \ldots, s_{k-r+1}},
$$

cf. formula (3.7). For $j \in J$, we define the operator $K_{j}(x): \mathcal{O}\left(C_{\mathcal{C}(x), a}\right) \rightarrow \mathcal{O}\left(C_{\mathcal{C}(x), a}\right)$ by the formula

$$
K_{j}(x)=\sum_{C \in \mathfrak{C}} \frac{\lambda_{j}^{C}}{f_{C}(x)} L_{C},
$$

cf. formula (3.5).

Corollary 3.8 If the pair $(\mathcal{C}(x), a)$ is unbalanced for $x \in \mathbb{C}^{n}-\Delta$, then the operator of multiplication $\left[\frac{a_{j}}{f_{j}}\right] *_{x}$ in $\mathcal{O}\left(C_{\mathcal{C}(x), a}\right)$ equals the operator $K_{j}(x): \mathcal{O}\left(C_{\mathcal{C}(x), a}\right) \rightarrow$ $\mathcal{O}\left(C_{\mathcal{C}(x), a}\right)$ defined in (3.20).

\subsection{Corollary of Theorem 3.7}

For a section $I=\sum_{j_{1}, \ldots, j_{k}} I^{j_{1}, \ldots, j_{k}} w_{j_{1}, \ldots, j_{k}}$ of the bundle of algebras (3.16) and $j \in J$, we define $\frac{\partial I}{\partial z_{j}}=\sum \frac{\partial I^{j_{1}, \ldots, j_{k}}}{\partial z_{j}} w_{j_{1}, \ldots, j_{k}}$. This formula defines the combinatorial connection on the bundle of algebras (3.16).

Theorem 3.9 (Varchenko 2015) If a section I of the bundle of algebras (3.16) is flat with respect to the Gauss-Manin connection, then it satisfies the differential equations 


$$
\kappa \frac{\partial I}{\partial z_{j}}(x)=\left[\frac{a_{j}}{f_{j}}\right] *_{x} I(x), \quad j \in J .
$$

Notice that solutions of these differential equations are given by the hypergeometric integrals

$$
I_{\gamma}(x)=\sum_{\substack{\text { independent } \\\left\{j_{1}<\cdots<j_{k}\right\} \subset J}} I_{\gamma}^{j_{1}, \ldots, j_{k}}(x) w_{j_{1}, \ldots, j_{k}},
$$

where $\gamma(x) \in H_{k}\left(U(\mathcal{C}(x)),\left.\mathcal{L}_{\kappa}\right|_{U(\mathcal{C}(x))}\right)$ is a flat section of the Gauss-Manin connection on the homology bundle and $I_{\gamma}^{j_{1}, \ldots, j_{k}}(x)=\int_{\gamma(x)} e^{\Phi_{\mathcal{C}, a} / \kappa} \omega_{j_{1}} \wedge \cdots \wedge \omega_{j_{k}}$.

Notice the similarities between the differential equations in (3.21) and the standard differential equations associated with Frobenius structures, see Dubrovin (1996), Manin (1999).

\section{Langrangian Variety and Critical Set}

\subsection{Lagrangian Variety}

Consider $\mathbb{C}^{n}$ with coordinates $q_{1}, \ldots, q_{n}$ and the dual space $\left(\mathbb{C}^{n}\right)^{*}$ with the dual coordinates $p_{1}, \ldots, p_{n}$. The space $\mathbb{C}^{n} \times\left(\mathbb{C}^{n}\right)^{*}$ has symplectic form $\omega=\sum_{j=1}^{n} d p_{j} \wedge d q_{j}$. Two functions $M, N$ on $\mathbb{C}^{n} \times\left(\mathbb{C}^{n}\right)^{*}$ are in involution if $\{M, N\}=\sum_{j=1}^{n}\left(\frac{\partial M}{\partial q_{j}} \frac{\partial N}{\partial p_{j}}-\right.$ $\left.\frac{\partial M}{\partial p_{j}} \frac{\partial N}{\partial q_{j}}\right)=0$.

For a $k$-dimensional vector subspace $Y \subset \mathbb{C}^{n}$, denote by $Y^{\perp} \subset\left(\mathbb{C}^{n}\right)^{*}$ the annihilator of $Y$. The $n$-dimensional vector space $Y \times Y^{\perp}$ is a Lagrangian subspace of $\mathbb{C}^{n} \times\left(\mathbb{C}^{n}\right)^{*}$ with defining equations

$$
\begin{aligned}
F_{\alpha} & :=\sum_{j=1}^{n} \alpha_{j} p_{j}=0, \quad \alpha=\left(\alpha_{1}, \ldots, \alpha_{n}\right) \in Y, \\
G_{\beta} & :=\sum_{j=1}^{n} \beta_{j} q_{j}=0, \quad \beta=\left(\beta_{1}, \ldots, \beta_{n}\right) \in Y^{\perp} .
\end{aligned}
$$

The set of all functions $\left\{F_{\alpha}, G_{\beta}\right\}$ is in involution.

Fix a weight $a \in\left(\mathbb{C}^{\times}\right)^{n}$. Consider the invertible rational symplectic map $r_{a}$ : $\mathbb{C}^{n} \times\left(\mathbb{C}^{n}\right)^{*} \rightarrow \mathbb{C}^{n} \times\left(\mathbb{C}^{n}\right)^{*}$,

$$
\left(q_{1}, \ldots, q_{n}, p_{1}, \ldots, p_{n}\right) \mapsto\left(q_{1}+a_{1} / p_{1}, \ldots, q_{n}+a_{n} / p_{n}, p_{1}, \ldots, p_{n}\right)
$$

Denote

$$
L_{Y, a}=r_{a}\left(Y \times Y^{\perp}\right) \subset \mathbb{C}^{n} \times\left(\mathbb{C}^{n}\right)^{*}
$$


Lemma 4.1 Assume that for every $j \in J$, the subspace $Y$ does not lie in the hyperplane $q_{j}=0$ and the subspace $Y^{\perp}$ does not lie in the hyperplane $p_{j}=0$, then $L_{Y, a}$ is an irreducible smooth $n$-dimensional Lagrangian subvariety in $\mathbb{C}^{n} \times\{y \in$ $\left.\left(\mathbb{C}^{n}\right)^{*} \mid \prod_{j=1}^{n} p_{j}(y) \neq 0\right\}$ defined by equations

$$
\begin{aligned}
F_{\alpha} & :=\sum_{j=1}^{n} \alpha_{j} p_{j}=0, & \alpha=\left(\alpha_{1}, \ldots, \alpha_{n}\right) \in Y, \\
G_{\beta, a}: & : \sum_{j=1}^{n} \beta_{j}\left(q_{j}-a_{j} / p_{j}\right)=0, & \beta=\left(\beta_{1}, \ldots, \beta_{n}\right) \in Y^{\perp} .
\end{aligned}
$$

The set of all functions $\left\{F_{\alpha}, G_{\beta, a}\right\}$ is in involution.

Let $I=\left\{i_{1}, \ldots, i_{k}\right\} \subset J$ be a $k$-element subset and $\bar{I}$ its complement.

Lemma 4.2 Under hypotheses of Lemma 4.1, assume that I is such that the functions $q_{I}=\left\{q_{i} \mid i \in I\right\}$ form a coordinate system on $Y$. Then the functions $q_{I}$ and $p_{\bar{I}}=$ $\left\{p_{j} \mid j \in \bar{I}\right\}$ form a coordinate system on $L_{Y, a}$.

Proof The functions $p_{I}$ are expressed in terms of $p_{\bar{I}}$ with the help of equations $F_{\alpha}=0$. The functions $q_{\bar{I}}$ are expressed in terms of $q_{I}, p_{\bar{I}}$ with the help of equations $G_{\beta, a}=0$. Clearly the functions $q_{I}, p_{\bar{I}}$ are independent.

We order the functions of the coordinate system $q_{I}, p_{\bar{I}}$ according to the increase of the index. For example, if $k=3, n=6, I=\{1,3,6\}$, then the order is $q_{1}, p_{2}, q_{3}, p_{4}, p_{5}, q_{6}$.

Fix a basis $b^{i}=\left(b_{1}^{i}, \ldots, b_{n}^{i}\right), i=1, \ldots, k$, of $Y$. Let $t=\left(t_{1}, \ldots, t_{k}\right)$ be the associated coordinate system on $Y$. Then $\left.q_{j}\right|_{Y}=\sum_{i=1}^{k} b_{j}^{i} t_{i}$. For $I=\left\{i_{1}, \ldots, i_{k}\right\} \subset$ $J$, we denote $d_{I}=d_{i_{1}, \ldots, i_{k}}=\operatorname{det}_{i, l=1}^{k}\left(b_{i_{l}}^{i}\right)$, cf. Sect. 2.9.

Lemma 4.3 Let $I=\left\{i_{1}, \ldots, i_{k}\right\}$ and $I^{\prime}=\left\{i_{1}^{\prime}, \ldots, i_{k}^{\prime}\right\}$ be subsets of $J$ each satisfying the hypotheses of Lemma 4.2. Consider the corresponding ordered coordinate systems $q_{I}, p_{\bar{I}}$ and $q_{I^{\prime}}, p_{\bar{I}^{\prime}}$ on $L_{Y, a}$. Express the coordinates of the second system in terms of coordinates of the first system and denote by $\operatorname{Jac}_{I, \bar{I}^{\prime}}\left(q_{I}, p_{\bar{I}}\right)$ the Jacobian of this change. Then

$$
\operatorname{Jac}_{I, \bar{I}^{\prime}}\left(q_{I}, p_{\bar{I}}\right)=\left(d_{i_{1}^{\prime}, \ldots, i_{k}^{\prime}} / d_{i_{1}, \ldots, i_{k}}\right)^{2} .
$$

Proof This statement is proved in Varchenko (2015, Lemma 5.4) under the assumption that $Y \subset \mathbb{C}^{n}$ is a generic subspace with respect to the coordinate system $q_{1}, \ldots, q_{n}$. This implies the lemma since the left- and right-hand sides of the formula continuously depend on $Y$.

Let $I=\left\{i_{1}, \ldots, i_{k}\right\} \subset J$ satisfy the hypotheses of Lemma 4.3 , and $q_{I}, p_{\bar{I}}$ the corresponding ordered coordinate system on $L_{Y, a}$. The functions $q_{1}, \ldots, q_{n}$ form an ordered coordinate system on $\mathbb{C}^{n}$. Let $\pi_{L_{Y, a}}: L_{Y, a} \rightarrow \mathbb{C}^{n}$ be the restriction to $L_{Y, a}$ of the natural projection $\mathbb{C}^{n} \times\left(\mathbb{C}^{n}\right)^{*} \rightarrow \mathbb{C}^{n}$. Let $\operatorname{Jac}_{I}\left(q_{I}, p_{\bar{I}}\right)$ be the Jacobian of $\pi_{L_{Y, a}}$ with respect to the chosen coordinate systems. 
Theorem 4.4 The function $d_{I}^{2} \operatorname{Jac}_{I}$ on $L_{Y, a}$ does not depend on the choice of I and

$$
d_{I}^{2} \operatorname{Jac}_{I}=(-1)^{n-k} \sum_{M \subset J,|M|=n-k} d_{\bar{M}}^{2} \prod_{j \in M} \frac{a_{j}}{p_{j}^{2}} .
$$

Proof The function $d_{I}^{2} \mathrm{Jac}_{I}$ does not depend on $I$ by Lemma 4.3. Formula (4.4) is proved in Varchenko (2015, Theorem 3.8) under the assumption that $Y \subset \mathbb{C}^{n}$ is a generic subspace with respect to the coordinate system $q_{1}, \ldots, q_{n}$. This implies the theorem since the left- and right-hand sides of the formula continuously depend on $Y$.

\subsection{Fibers of $\pi_{L_{Y, a}}$}

For $x \in \mathbb{C}^{n}$, denote $C_{Y, a}(x)=\pi_{L_{Y, a}}^{-1}(x)$, the fiber of the projection $\pi_{L_{Y, a}}$. The fiber is defined in $\left(\mathbb{C}^{\times}\right)^{n}$ with coordinates $p_{1}, \ldots, p_{n}$ by the equations

$$
\begin{aligned}
\sum_{j=1}^{n} \alpha_{j} p_{j} & =0, \quad \alpha=\left(\alpha_{1}, \ldots, \alpha_{n}\right) \in Y, \\
\sum_{j=1}^{n} \beta_{j}\left(x_{j}-a_{j} / p_{j}\right) & =0, \quad \beta=\left(\beta_{1}, \ldots, \beta_{n}\right) \in Y^{\perp},
\end{aligned}
$$

cf. (4.3). Let $I_{L_{Y}(x), a}$ be the ideal in $\mathcal{O}\left(\left(\mathbb{C}^{\times}\right)^{n}\right)$ generated by the left-hand sides of equations (4.5). Set

$$
\mathcal{O}\left(L_{Y, a}(x)\right)=\mathcal{O}\left(\left(\mathbb{C}^{\times}\right)^{n}\right) / I_{L_{Y}(x), a}
$$

\subsection{Arrangement in $\mathbb{C}^{n} \times \mathbb{C}^{k}$}

Return to the objects and notations of Sect. 3 and consider $\mathbb{C}^{k}$ with coordinates $t_{1}, \ldots, t_{k}, \mathbb{C}^{n}$ with coordinates $z_{1}, \ldots, z_{n}$, the projection $\pi: \mathbb{C}^{n} \times \mathbb{C}^{k} \rightarrow \mathbb{C}^{n}$ and $n$ nonzero linear functions on $\mathbb{C}^{k}, g_{j}=b_{j}^{1} t_{1}+\cdots+b_{j}^{k} t_{k}, j \in J$. As in Sect. 3 we assume that $g_{j}, j \in J$, span the dual space $\left(\mathbb{C}^{k}\right)^{*}$. We consider the linear functions $f_{j}=g_{j}+z_{j}=b_{j}^{1} t_{1}+\cdots+b_{j}^{k} t_{k}+z_{j}$ on $\mathbb{C}^{n} \times \mathbb{C}^{k}$ and the arrangement of hyperplanes $\mathcal{C}=\left\{H_{j}\right\}_{j \in J}$ in $\mathbb{C}^{n} \times \mathbb{C}^{k}$, where $H_{j}$ is defined by the equation $f_{j}=0$. We assume that a weight $a \in\left(\mathbb{C}^{n}\right)^{\times}$is given and consider the critical set $C_{\mathcal{C}, a}$ defined by (3.14). In the rest of the paper we denote by

$$
Y=Y(\mathcal{C})
$$

the $k$-dimensional subspace of $\mathbb{C}^{n}$ spanned by the vectors $b^{i}=\left(b_{1}^{i}, \ldots, b_{n}^{i}\right), i=$ $1, \ldots, k$.

Theorem 4.5 Assume that for any $j \in J$, the subspace $Y^{\perp}$ does not lie in the hyperplane $p_{j}=0$. Assume that the critical set $C_{\mathcal{C}, a}$ is nonempty. Then the map 


$$
\Psi_{\mathcal{C}, a}: U(\mathcal{C}) \rightarrow \mathbb{C}^{n} \times\left(\mathbb{C}^{n}\right)^{*}, \quad(x, u) \mapsto(x, y(x, u)),
$$

where

$$
y(x, u)=\left(\frac{\partial \Phi_{\mathcal{C}, a}}{\partial z_{1}}(x, u), \ldots, \frac{\partial \Phi_{\mathcal{C}, a}}{\partial z_{n}}(x, u)\right)=\left(\frac{a_{1}}{f_{1}(x, u)}, \ldots, \frac{a_{n}}{f_{n}(x, u)}\right)
$$

restricted to $C_{\mathcal{C}, a}$ is a diffeomorphism of the critical set $C_{\mathcal{C}, a}$ onto the Lagrangian variety $L_{Y, a}$.

Proof .

Lemma 4.6 We have $\Psi_{\mathcal{C}, a}\left(C_{\mathcal{C}, a}\right) \subset L_{Y, a}$.

Proof If $\alpha \in Y$ and $(x, u) \in C_{\mathcal{C}, a}$, then the equation $F_{\alpha}\left(\frac{\partial \Phi_{\mathcal{C}, a}}{\partial z_{1}}(x, u), \ldots, \frac{\partial \Phi_{\mathcal{C}, a}}{\partial z_{n}}(x, u)\right)$ $=0$ is a linear combination of the equations $\frac{\partial \Phi_{\mathcal{C}, a}}{\partial t_{i}}(x, u)=0, i=1, \ldots, k$, see (2.7). If $\beta \in Y^{\perp}$ and $(x, u) \in C_{\mathcal{C}, a}$, then the equation $G_{\beta, a}\left(x, \frac{\partial \Phi_{\mathcal{C}, a}}{\partial z_{1}}(x, u), \ldots, \frac{\partial \Phi_{\mathcal{C}, a}}{\partial z_{n}}(x, u)\right)$ $=0$ is just the Eq. (3.1).

Lemma 4.7 The map $\Psi_{\mathcal{C}, \text { a }}$ sends distinct points of $U(\mathcal{C})$ to distinct points of $\mathbb{C}^{n} \times$ $\left(\mathbb{C}^{n}\right)^{*}$.

Proof It is enough to check that $\Psi_{\mathcal{C}, a}(x, u) \neq \Psi_{\mathcal{C}, a}\left(x, u^{\prime}\right)$ if $u \neq u^{\prime}$, but this follows from Lemma 2.6.

Lemma 4.8 The Jacobian of the map $\left.\Psi_{\mathcal{C}, a}\right|_{C_{\mathcal{C}, a}}: C_{\mathcal{C}, a} \rightarrow L_{Y, a}$ is never zero.

Proof The lemma follows from Lemmas 4.1, 3.5, 4.7 or by direct calculation.

Lemma 4.9 We have $\Psi_{\mathcal{C}, a}\left(C_{\mathcal{C}, a}\right)=L_{Y, a}$.

Proof Let $\Psi_{\mathcal{C}, a}\left(C_{\mathcal{C}, a}\right) \neq L_{Y, a}$ and $\left(x^{0}, y^{0}\right) \in L_{Y, a}-\Psi_{\mathcal{C}, a}\left(C_{\mathcal{C}, a}\right)$, where $x^{0} \in \mathbb{C}^{n}$ and $y^{0} \in\left(\mathbb{C}^{n}\right)^{*}$. We have $\operatorname{dim}\left(L_{Y, a}-\Psi_{\mathcal{C}, a}\left(C_{\mathcal{C}, a}\right)\right)<n$ by Lemmas $4.1,3.5,4.8$. Hence there exists a germ of an analytic curve $\iota:(\mathbb{C}, 0) \rightarrow\left(L_{Y, a},\left(x^{0}, y^{0}\right)\right)$ such that $\iota(s) \in \Psi_{\mathcal{C}, a}\left(C_{\mathcal{C}, a}\right)$ for $s \neq 0$. Consider the curve $s \mapsto\left(\Psi_{\mathcal{C}, a}\right)^{-1}(\iota(s))$ for $s \neq 0$. Let $\left(\Psi_{Y, a}\right)^{-1}(\iota(s))=(x(s), u(s))$, where $x(s) \in \mathbb{C}^{n}$ and $u(s) \in \mathbb{C}^{k}$. We have $\lim _{s \rightarrow 0} \gamma(s)=x^{0}$. Since for any $j \in J$, the function $\frac{\partial \Phi_{\mathcal{C}, a}}{\partial z_{j}}(x(s), y(s))$ has a finite limit, there is a finite nonzero limit $u^{0}:=\lim _{s \rightarrow 0} u(s)$ in $U\left(\mathcal{C}\left(x^{0}\right)\right)$. Clearly $\left(x^{0}, u^{0}\right) \in C_{\mathcal{C}, a}$ and $\Psi_{\mathcal{C}, a}:\left(x^{0}, u^{0}\right) \mapsto\left(x^{0}, y^{0}\right)$. We get a contradiction which proves the lemma.

One can also check the statement by direct calculation.

Lemmas 4.6, 4.7, 4.8 and 4.9 prove Theorem 4.5. 


\subsection{Hessian and Jacobian}

Recall the Hessian of the master function $\Phi_{\mathcal{C}, a}$, see Sect. 2.10. Under the diffeomorphism $C_{\mathcal{C}, a} \rightarrow L_{Y, a}$ of Theorem 4.5 , we may consider the Hessian as a function on $L_{Y, a}$. Then

$$
\operatorname{Hess}_{\mathcal{C}, a}=(-1)^{k} \sum_{I \subset J,|I|=k} d_{I}^{2} \prod_{i \in I}^{k} \frac{p_{i}^{2}}{a_{i}}
$$

Corollary 4.10 Let $I \subset J$ satisfy the hypotheses of Lemma 4.2. Then

$$
\operatorname{Hess}_{\mathcal{C}, a}=(-1)^{n} d_{I}^{2} \operatorname{Jac}_{I} \prod_{j \in J} \frac{p_{j}^{2}}{a_{j}}
$$

Proof Formula (4.11) follows from formulas (4.4) and (4.10).

Notice that the ratio of $\operatorname{Hess}_{\mathcal{C}, a}$ and $d_{I}^{2} \mathrm{Jac}_{I}$ is never zero.

\subsection{Corollaries of Theorem 4.5}

The map $\Psi_{\mathcal{C}, a}$ of Theorem 4.5 establishes an isomorphism

$$
\Psi_{\mathcal{C}, a}^{*}: \mathcal{O}\left(L_{Y, a}\right) \rightarrow \mathcal{O}\left(C_{\mathcal{C}, a}\right), \quad\left[q_{j}\right], \mapsto\left[z_{j}\right], \quad\left[p_{j}\right] \mapsto\left[\frac{\partial \Phi_{\mathcal{C}, a}}{\partial z_{j}}\right],
$$

for all $j \in J$, and for any $x \in \mathbb{C}^{n}$, the isomorphism

$$
\Psi_{\mathcal{C}(x), a}^{*}: \mathcal{O}\left(L_{Y, a}(x)\right) \rightarrow \mathcal{O}\left(C_{\mathcal{C}(x), a}\right), \quad\left[p_{j}\right] \mapsto\left[\frac{\partial \Phi_{\mathcal{C}, a}}{\partial z_{j}}\right], \quad j \in J .
$$

In particular, if the weight $a$ is such that $(\mathcal{C}(x), a)$ is unbalanced, then the number of solutions of system (4.5), counted with multiplicities, equals $|U(\mathcal{C}(x))|$ and can be calculated in terms of the matroid associated with the arrangement $\mathcal{C}(x)$, see Lemma 3.6.

The isomorphism $\Psi_{\mathcal{C}(x), a}^{*}$ allows us to compare objects associated with $\mathcal{O}\left(C_{\mathcal{C}(x), a}\right)$ and objects associated with $\mathcal{O}\left(L_{Y, a}(x)\right)$. For example, let $q_{I}, p_{\bar{I}}$ be an ordered coordinate system on $L_{Y, a}$ like in Lemma 4.2. Assume that $x \in \mathbb{C}^{n}$ is such that $\operatorname{dim} \mathcal{O}\left(L_{Y, a}(x)\right)$ is finite. Consider the Grothendieck residue $\mathcal{R}: \mathcal{O}\left(L_{Y, a}(x)\right) \rightarrow \mathbb{C}$,

$$
[f] \mapsto \frac{1}{(2 \pi i)^{n}} \int_{\Gamma} \frac{f d p_{I} \wedge d q_{\bar{I}}}{\prod_{j=1}^{n} q_{j}}
$$

where the differentials are ordered as in the ordered coordinate system $q_{I}, p_{\bar{I}}$, cf. (2.9). Define the nondegenerate bilinear form $(,)_{L_{Y, a}(x)}$ on $\mathcal{O}\left(L_{Y, a}(x)\right)$ by the formula 


$$
([f],[g])_{L_{Y, a}(x)}=\frac{(-1)^{n}}{d_{I}^{2}} \mathcal{R}\left([f][g] \prod_{j=1}^{n} \frac{a_{j}}{\left[p_{j}^{2}\right]}\right) .
$$

Corollary 4.11 Assume that the weight a is generic in the sense of Lemma 3.6, then the isomorphism $\Psi_{\mathcal{C}(x), a}^{*}$ identifies the form $(,)_{L_{Y, a}(x)}$ on $\mathcal{O}\left(L_{Y, a}(x)\right)$ and the form $(,)_{C_{\mathcal{C}(x), a}(x)}$ on $\mathcal{O}\left(C_{\mathcal{C}(x), a}\right)$.

Proof For generic $x \in \mathbb{C}^{n}$, the corollary follows from Corollary 4.10. For all $x \in \mathbb{C}^{n}$, the corollary follows by continuity.

Remark. Notice that the form $(,)_{L_{Y, a}(x)}$ is given by an $n$-dimensional integral while the form $(,)_{C_{\mathcal{C}(x), a}(x)}$ is given by a $k$-dimensional integral.

If $(\mathcal{C}(x), a)$ is unbalanced for some $x \in \mathbb{C}^{n}-\Delta$, then we have the vector bundle of algebras

$$
\sqcup_{x \in \mathbb{C}^{n}-\Delta} \mathcal{O}\left(L_{Y, a}(x)\right) \rightarrow \mathbb{C}^{n}-\Delta
$$

The fiber isomorphism $\Psi_{\mathcal{C}(x), a}^{*}$ identifies this bundle of algebras with the bundle of algebras in (3.16). The combinatorial and Gauss-Manin connections on the bundle of algebras in (3.16) induce the corresponding connections on the bundle in (4.16).

For $x \in \mathbb{C}^{n}-\Delta$, we define the marked elements $p_{i_{1}, \ldots, i_{k}}$ in $\mathcal{O}\left(L_{Y, a}(x)\right)$ as the images under $\Psi_{\mathcal{C}(x), a}^{*}$ of the marked elements $w_{i_{1}, \ldots, i_{k}}$ in $\mathcal{O}\left(C_{\mathcal{C}(x), a}\right)$. By formula (2.30), we have

$$
p_{i_{1}, \ldots, i_{k}}=d_{i_{1}, \ldots, i_{k}} a_{i_{1}} \ldots a_{i_{k}} p_{i_{1}} \ldots p_{i_{k}}
$$

with the skew-symmetry property

$$
p_{i_{\sigma(1)}, \ldots, i_{\sigma(k)}}=(-1)^{\sigma} p_{i_{1}, \ldots, i_{k}}, \quad \text { for } \sigma \in \Sigma_{k}
$$

and the marked relations

$$
\sum_{j \in J} p_{j, i_{2}, \ldots, i_{k}}=0
$$

labeled by independent subsets $\left\{i_{2}, \ldots, i_{k}\right\}$. By Corollary (2.18), the marked elements $p_{i_{1}, \ldots, i_{k}}$ span $\mathcal{O}\left(L_{Y, a}(x)\right)$ as a vector space and relations (4.18), (4.19) are the only linear relations between them. This fact defines an integral structure on $\mathcal{O}\left(L_{Y, a}(x)\right)$.

For a section $I=\sum_{j_{1}, \ldots, j_{k}} I^{j_{1}, \ldots, j_{k}} p_{j_{1}, \ldots, j_{k}}$ of the bundle of algebras (4.16) and $j \in J$, we define $\frac{\partial I}{\partial q_{j}}=\sum \frac{\partial I^{j_{1}, \ldots, j_{k}}}{\partial q_{j}} p_{j_{1}, \ldots, j_{k}}$. This formula defines the combinatorial connection on (4.16).

Theorem 4.12 If a section I of the bundle of algebras (4.16) is flat with respect to the Gauss-Manin connection, then it satisfies the differential equations 


$$
\kappa \frac{\partial I}{\partial q_{j}}(x)=\left[p_{j}\right] *_{x} I(x), \quad j \in J,
$$

where $\left[p_{j}\right] *_{x}$ is the operator of multiplication by $\left[p_{j}\right]$ in $\mathcal{O}\left(L_{Y, a}(x)\right)$.

Remark. Notice that solutions of these differential equations are given by the multidimensional hypergeometric integrals as in (3.22). Notice also the action of $\left[p_{j}\right] *_{x}$ on the marked elements $p_{i_{1}, \ldots, i_{k}}$ can be identified with action on the marked elements $w_{i_{1}, \ldots, i_{k}}$ of the operator $K_{j}(x)$ from (3.20).

\subsection{Real Solutions}

Assume that for any $j \in J$, the subspace $Y^{\perp}$ does not lie in the hyperplane $p_{j}=0$. Assume that all coordinates of the weight $a \in\left(\mathbb{C}^{\times}\right)^{n}$ are positive. Assume that all entries of the matrix $\left(b_{j}^{i}\right)_{\substack{i=1, \ldots, k \\ j=1, \ldots, n}}$, defining $Y \subset \mathbb{C}^{n}$ in (4.7), are real. Assume that the critical set $C_{\mathcal{C}, a}$ is nonempty.

Corollary 4.13 Under these assumptions, if $x \in \mathbb{R}^{n} \subset \mathbb{C}^{n}$, then all solutions of system (4.5) are real and nondegenerate, and the number of solutions equals $\mid \chi(U(\mathcal{C}(x)) \mid$.

Proof If $a \in\left(\mathbb{R}_{>0}\right)^{n}, x \in \mathbb{R}^{n}$, and $\left(b_{j}^{i}\right)$ are real, then all points of the critical set $C_{\mathcal{C}(x), a}$ are real, nondegenerate, and the number of points equals $\mid \chi(U(\mathcal{C}(x)) \mid$, see Varchenko (1995). Now the corollary follows from Theorem 4.5.

The reality property in Corollary 4.13 is similar to the reality property of Schubert calculus, see Mukhin et al. (2009a), Mukhin et al. (2009b), Sottile (2010).

\section{Characteristic Variety of the Gauss-Manin Differential Equations}

Consider the Gauss-Manin differential equations $\kappa \frac{\partial I}{\partial z_{j}}=K_{j} I$ in (3.13). Define the characteristic variety of the $\kappa$-dependent $D$-module associated with the Gauss-Manin differential equations as

$$
\operatorname{Spec}_{\mathcal{C}, a}=\left\{(x, y) \in\left(\mathbb{C}^{n}-\Delta\right) \times\left(\mathbb{C}^{n}\right)^{*} \mid \exists v \in \operatorname{Sing}_{a} V \text { with } K_{j}(x) v=y_{j} v, j \in J\right\} .
$$

Let $\pi_{\operatorname{Spec}_{\mathcal{C}, a}}: \operatorname{Spec}_{\mathcal{C}, a} \rightarrow \mathbb{C}^{n}$ be the projection to $\mathbb{C}^{n}$.

Recall the Lagrangian variety $L_{Y, a} \subset \mathbb{C}^{n} \times\left(\mathbb{C}^{n}\right)^{*}$ introduced in Sect. 4.3 and the projection $\pi_{L_{Y, a}}: L_{Y, a} \rightarrow \mathbb{C}^{n}$.

Theorem 5.1 Assume that the weight a is generic in the sense of Lemma 3.6, then $\operatorname{Spec}_{\mathcal{C}, a}=\pi_{L_{Y, a}}^{-1}\left(\mathbb{C}^{n}-\Delta\right)$.

Proof For generic $x \in \mathbb{C}^{n}-\Delta$, the special vectors $(\mathrm{F}(u))_{u \in C_{\mathcal{C}, a}}$ form a basis of Sing ${ }_{a} V$ by Theorem 2.11. This gives $\pi_{\operatorname{Spec}_{\mathcal{C}, a}}^{-1}(x)=\pi_{L_{Y, a}}^{-1}(x)$ by Theorems 2.12 and 3.7. We get the equality $\pi_{\operatorname{Spec}_{\mathcal{C}, a}}^{-1}(x)=\pi_{L_{Y, a}}^{-1}(x)$ for all $x \in \mathbb{C}^{n}-\Delta$ by continuity.

Theorem 5.1 is proved in Varchenko (2015) if $\mathcal{C}$ is generic. 
Acknowledgments The author thanks V. Tarasov for collaboration. The proof of Theorem 2.16 is the result of joint efforts. The author thanks B. Dubrovin and A. Veselov for helpful discussions.

\section{References}

Arnol'd, V.I., Gusein-Zade, S.M., Varchenko, A.N.: Singularities of Differential Maps, vol. I. Nauka, Moscow (1982) (also Birkhauser, 1985)

Bayer, M., Brandt, K.: Discriminantal arrangements, fiber polytopes and formality. J. Algebr. Comb. 6(3), 229-246 (1997)

Cohen, D., Orlik, P.: Stratified Morse theory in arrangements. Pure Appl. Math. Q. 2 3(1), 673-697 (2006)

Crapo, H.: A higher invariants for matroids. J. Comb. Theory 2, 406-417 (1967)

Dubrovin B.: Geometry of 2D topological field theories. In: Francaviglia, M., Greco, S. (eds.) Integrable Systems and Quantum Groups. Lecture Notes in Mathematics, vol. 1620, pp. 120-348. Springer (1996)

Etingof, P., Frenkel, I., Kirillov, A.: Lectures on Representation Theory and Knizhnik-Zamolodchikov Equations, Mathematical Surveys and Monographs, p. 58. AMS (1998)

Falk, M., Varchenko, A.: The contravariant form on singular vectors of a projective arrangement. In: Bjorner, A., Cohen, F., De Concini, C., Procesi, C., Salvetti, M. (eds.) Configuration Spaces, Geometry, Combinatorics and Topology, pp. 255-272. CRM Series (2012)

Givental, A.: Stationary phase integrals, quantum Toda lattices, flag manifolds and the mirror conjecture, topics in singularity theory. AMS Transl. Ser. 2(180), 103-115 (1997)

Griffiths, P., Harris, J.: Principles of Algebraic Geometry. Wiley, New York (1994)

Givental, A., Kim, B.: Quantum cohomology of flag manifolds and Toda lattices. Commun. Math. Phys. 168(3), 455-675 (1995)

Gelfand, I.M., Varchenko, A.: On Heaviside functions of a configuration of hyperplanes. Funk. Anal. i Prilozh. 21(4), 1-18 (1987). (p. 96)

Manin, Y.I.: Frobenius Manifolds, Quantum Cohomology, and Moduli Spaces, vol. 47. American Mathematical Society Colloquium Publications (AMS), Providence (1999)

Mukhin, E., Tarasov, V., Varchenko, A.: Bethe algebra of the $\mathfrak{g l}_{N+1}$ Gaudin model and algebra of functions on the critical set of the master function. In: New Trends in Quantum Integrable Systems, pp. 307-324. World Scientific Publication, Hackensack (2011)

Mukhin, E., Tarasov, V., Varchenko, A.: The B. and M. Shapiro conjecture in real algebraic geometry and the Bethe ansatz. Ann. Math. (2) 170(2), 863-881 (2009)

Mukhin, E., Tarasov, V., Varchenko, A.: Schubert calculus and representations of the general linear group. J. Am. Math. Soc. 22(4), 909-940 (2009)

Mukhin, E., Tarasov, V., Varchenko, A.: KZ characteristic variety as the zero set of classical Calogero-Moser Hamiltonians. SIGMA 8, 11 (2012). (Paper 072)

Mukhin, E., Varchenko, A.: Norm of a Bethe vector and the Hessian of the master function. Compos. Math. 141(4), 1012-1028 (2005)

Orlik, P., Terao, H.: The number of critical points of a product of powers of linear functions. Invent. Math. 120(1), 1-14 (1995)

Orlik, P., Terao, H.: Arrangements and hypergeometric integrals, vol. 9, p. 112. MSJ Memoir. Math Soc, Japan, Tokyo, ix+ (2001)

Proudfoot, N.: The equivariant Orlik-Solomon algebra. J. Algebra 305(2), 1186-1196 (2006)

Silvotti, R.: On a conjecture of Varchenko. Invent. Math. 126(2), 235-248 (1996)

Sottile, F.: Frontiers of reality in Schubert calculus. Bull. Am. Math. Soc. (N.S.) 47(1), 31-71 (2010)

Schechtman, V., Terao, H., Varchenko, A.: Local systems over complements of hyperplanes and the KacKazhdan conditions for singular vectors. J. Pure. Appl. Algebra 100, 93-102 (1995)

Schechtman, V., Varchenko, A.: Arrangements of hyperplanes and Lie algebra homology. Invent. Math. 106, 139-194 (1991)

Tarasov, V., Varchenko, A.: Hypergeometric solutions of the quantum differential equation of the cotangent bundle of a partial flag variety. Cent. Eur. J. Math. 12(5), 694-710 (2014)

Varchenko, A.: Beta-function of Euler, Vandermonde determinant, Legendre equation and critical values of linear functions of configuration of hyperplanes, I. Izv. Akademii Nauk USSR, Seriya Mat. 53(6), 1206-1235 (1989) 
Varchenko, A.: Multidimensional hypergeometric functions and representation theory of Lie Algebras and Quantum Groups. In: Advanced Series in Mathematical Physics, vol. 21. World Scientific, River Edge (1995)

Varchenko, A.: Critical points of the product of powers of linear functions and families of bases of singular vectors. Compos. Math. 97, 385-401 (1995)

Varchenko, A.: Bethe Ansatz for arrangements of hyperplanes and the Gaudin Model. Mosc. Math. J. 6(1), 195-210 (2006). pp. 223-224

Varchenko, A.: Quantum integrable model of an arrangement of hyperplanes. Symmetry Integr. Geom. Methods Appl. 7, 55 (2011). (Paper 032)

Varchenko, A.: Arrangements and Frobenius like structures. Annales de la faculte des sciences de Toulouse Ser. 6 24(1), 133-204 (2015)

Varchenko, A.: Characteristic variety of the Gauss-Manin differential equations of a generic parallelly translated arrangement. Mathematics 2, 218-231 (2014)

Yuzvinsky, S.: Cohomology of the Brieskorn-Orlik-Solomon algebras. Commun. Algebra 23, 5339-5354 (1995) 